\title{
The Mathematical Modelling of Cell Kinetics in Corneal Epithelial Wound Healing
}

\author{
E. A. Gaffney*†, P. K. Maini*, J. A. Sherratt $\$$ and S. Tuft \\ *Centre for Mathematical Biology, Mathematical Institute, 24-29 St Giles', Oxford \\ $O X 13 L B, U . K ., \$ D e p a r t m e n t$ of Mathematics, Heriot-Watt University, Edinburgh \\ EH14 4AS, U.K. and §Moorfields Eye Hospital, 162 City Road, London EC1V 2PD, \\ $U . K$.
}

(Received on 17 June 1998, Accepted in revised form on 15 October 1998)

\begin{abstract}
This paper considers the comparison of experimental spatial and temporal data of mitotic rates measured during corneal epithelial wound healing $(\mathrm{CEWH})$ of a rat model with the predictions of a computer modelling framework. We begin by briefly showing that previous models, used in the study of corneal epithelial wound healing speeds, are inadequate for the study of cell kinetics. We proceed to formulate a new modelling framework more suited to such a study. This framework is simulated in its simplest form, and the results from this motivate a new realisation of the modelling framework, including a caricature of age structuring. Finally, a model with a simple representation of juxtacrine signalling is considered. The final model captures many, though not all, of the trends of the experimental data. This paper thus lays a foundation for the modelling of the cell kinetics of corneal epithelial wound healing, and yields valuable insight regarding the important mechanisms a model should consider in order to reproduce the observed experimental trends.
\end{abstract}

(C) 1999 Academic Press

\section{Introduction}

Evolutionary pressures have spurred the development of many complex wound healing mechanisms in higher organisms. Unique among such mechanisms is the highly specialised healing of the cornea. The overwhelming need to maintain visual function requires the absence of angiogenesis in corneal wound healing. Such considerations imply that corneal wound healing

$\dagger$ Author to whom correspondence should be addressed. E-mail: gaffney@maths.ox.ac.uk is of medical importance and, in many ways, relatively simple compared with other wound healing mechanisms. This is reflected by a substantial literature which increasingly includes quantitative data, especially in the specific aspect of corneal epithelial repair, which is the focus of this paper. For this subfield, there is also the promise of remarkably detailed observational insight into cell kinetics and cell dynamics, and perhaps large amounts of multidimensional quantitative data too, due to the recent development of confocal microscopy techniques 
for the in vivo corneal epithelium (Masters, 1995). Consequently mathematical and computational modelling, though currently in its infancy in this particular area, may reasonably be expected to play an ever increasing role in the coordination and development of a quantitative understanding of the corneal epithelium and its complex wound healing response.

In this paper the modelling of the corneal epithelium and its repair is considered within a simplified picture. Pathological possibilities such as angiogenesis and conjunctival invasion of the cornea are discounted, as are innervation, the immunological system and many other intrinsic biological features. One thus initially considers a system of epithelial cells in various states of differentiation on a basal lamina. The tissue dynamics are controlled by numerous types of cell-cell and cell-substrate interactions. These interactions, together with the cell proliferation and differentiation rates and extracellular matrix component synthesis rates are critically dependent on several different growth factors. There are many possible sources of these growth factors, including cell excretions, tear fluid, and in a wounded cornea, cell debris and possibly exogenous application.

Further complications arise from the structure of the cornea whose unwounded state is a dynamic equilibrium. Cells are continually shed from the corneal surface, and continually replaced by proliferation within the corneal epithelium. The stem cells, which represent the ultimate progenitor source of the corneal mass, are localised at the edge of the cornea, in a region referred to as the limbus. The stem cell population is, in non-pathological scenarios, self sustaining and also produces, via asymmetric cell division, transient amplifying cells (Tseng, 1989). These cells have a relatively short cell cycle time and can undergo numerous rounds of cell division. There is a continual flux of transient amplifying cells onto the peripheral areas of the cornea, where they migrate radially, undergo cell division, and differentiate to form post-mitotic cells (typically the wing and surface cells of the cornea). This results in an implicit spatial heterogeneity of the unwounded cornea, coupled to the age structure of the corneal epithelial cells, which can be a complicating factor in modelling

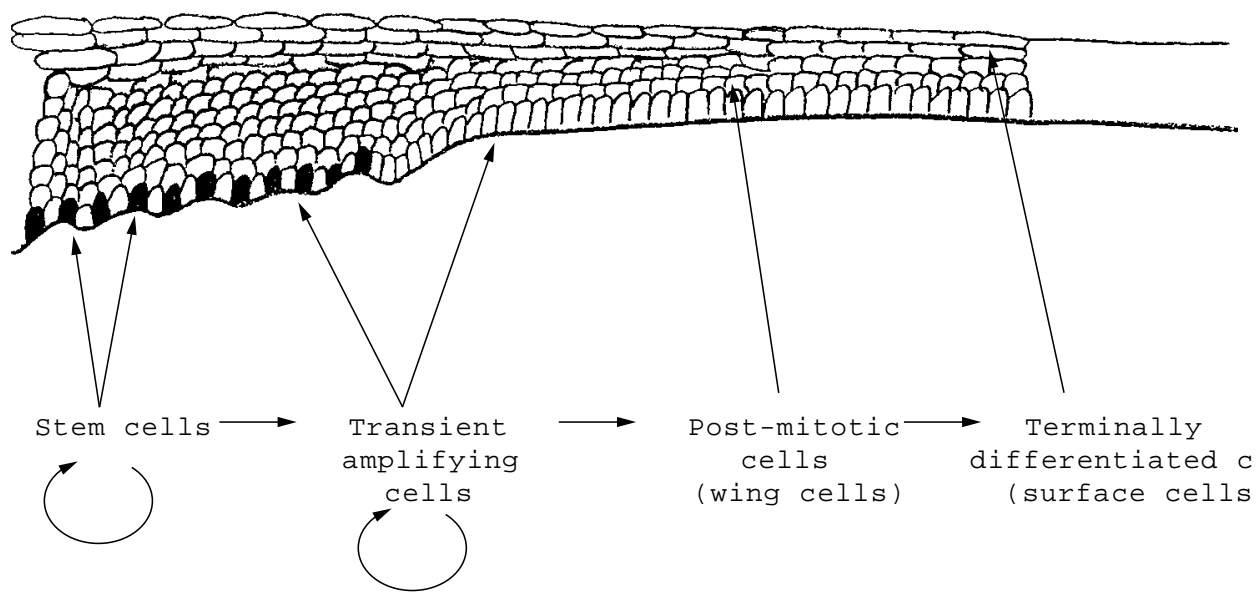

FIG. 1. A schematic diagram showing the correlation between the stages of cell differentiation and spatial position. The stem cells (black) are located in the basal layers of the limbus. The transient amplifying cells tend to be localised in the basal layers. The quiescent cells, namely the post-mitotic wing cells and the terminally differentiated surface cells, occur in the non-basal layers. Movement between the cell compartments is also indicated, via the horizontal arrows, while (symmetric) cell divisions are indicated by the looped "feedback" arrows. The diagram is based on Figs 7.5 and 8.2 of Bron et al. (1997) and Fig. 1 of Kruse (1994). 
studies, as depicted schematically in Fig. 1, which is reprinted at the end of the paper. This heterogeneity and age structure affects the wound healing response, as the proliferative resources are concentrated in the limbal and peripheral corneal regions.

Even the above simplified picture is too complex to be modelled; the modelling techniques are not currently powerful enough, and not enough is known about the corneal epithelium to attempt to incorporate such detail. Focusing on specifics within this picture has nonetheless been fruitful (reviewed in Gaffney et al., 1997). Simple mathematical and computational models of corneal epithelial wound healing (CEWH), developed by Dale, Sherratt and Maini (1994a, b), have been successful in predicting wound healing speeds. This model involved a single cell type and a single chemical stimulus, and considered how they would interact, leading to a phenomenological model of two coupled nonlinear partial differential equations. Investigating the wound fronts of this system, and its travelling wave analogue, led to predictions of wound healing speeds that agreed closely with experimental observations for biologically reasonable parameters.

The intention in this paper is to build on such foundations to investigate the modelling of cell kinetics in CEWH. It is particularly motivated by the publication of quantitative spatial and temporal experimental cell kinetic data (Sandvig \& Haaskjold, 1993; Sandvig et al., 1994) during CEWH of the rat model. Thus our overall aim is to investigate whether focusing on aspects of the above picture of CEWH can yield a modelling framework capable of capturing the trends of this published cell kinetic data.

In the next section below, the experimental cell kinetic data is briefly summarised. In the following section the Dale-Sherratt-Maini model mentioned above is used in an attempt to simulate the experimental cell kinetic data. This model however proves to be inadequate, but the form of this inadequacy clearly motivates the generalisations considered in this paper. Such considerations are used to construct a new modelling framework for CEWH, more capable of capturing the experimental data. We proceed to develop a simple realisation of the framework which is followed by a presentation of the modelling results. These indicate that the introduction of extra mechanisms in the model, including age structure effects, would be crucial for capturing the trends of the published data.

This motivates the development of two further realisations of the modelling framework, both of which incorporate a caricature of the effects of age structure. The predictions of these models are presented and discussed. The fact that many, though not all, of the trends in the experimental data are reproduced by the final model developed in this paper represents a substantial advance in the modelling of the cell kinetics of CEWH. This final model also indicates the type of mechanisms that may be needed to ensure agreement of modelling and experiment, which is crucial if future experimentation could yield data that could support a more sophisticated modelling effort, including details such as age structured dynamics.

\section{Preliminaries}

\subsection{EXPERIMENTAL RESULTS}

The modelling of this paper is motivated by the experimental measurement of mitotic rates for various times and at numerous positions for the repair of rat corneal epithelial wounds, as measured by Sandvig et al. (1993, 1994). Here we consider the results of three experiments; the first was presented in Sandvig \& Haaskjold (1993), while the remaining two were presented in Sandvig et al. (1994). The key observable to be compared with the model predictions is the measured mitotic rate. A summary of how this was measured is as follows. Four hours prior to sacrifice, rats were injected with a metaphase arrest agent (Colchicine) which arrested the cell cycle of any cell entering mitotic metaphase up to the point of sacrifice. The percentage of cells arrested in the mitotic metaphase after sacrifice, as determined by direct counting, was denoted to 

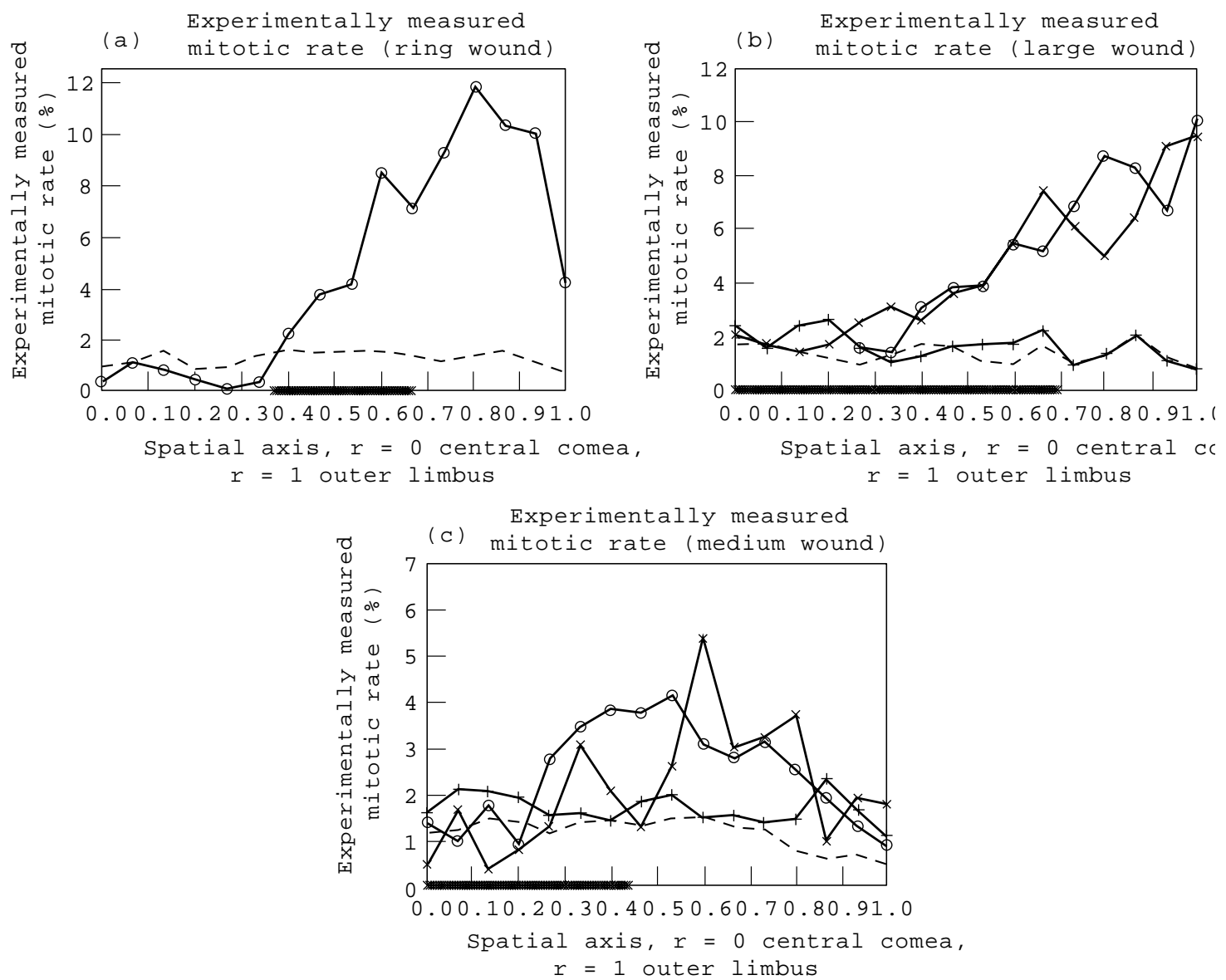

FIG. 2. Experimental plots of mitotic rates at various times following wounding of the rat corneal epithelium. The horizontal axes of the above graphs are scaled in terms of a typical rat corneal radius, which is approximately $0.4 \mathrm{~cm}$; each horizontal axis is also marked with a thick line, representing the initial wound size for the data given. In (a) the plots are a control (---) and the mitotic rate at 28 hours after wounding $(\bigcirc)$; (b) and (c) gives the mitotic rates at $24(\bigcirc), 48(\times)$ and $96(+)$ hr after wounding, plus control mitotic rates $(---)$. See text for full discussion. Replotted from Sandvig \& Haaskjold (1993) and Sandvig et al. (1994).

be the mitotic rate and plotted across the cornea for various times. The experimental researchers took care to prevent discrepancies between individual measurements due to circadian variation.

A summary of the quantitative experimental results has been reproduced for completeness in Fig. 2; the figures are labelled (a), (b) and (c) to enable future comparisons. Figure 2(a) represents the mitotic rate at 28 hours after wounding for an initial wound in the shape of a ring on the cornea, and also gives unwounded, control data. The paper by Sandvig \& Haaskjold (1993) should be consulted for more experimental details concerning these measurements.
Figure 2(b) gives the mitotic rates at 24, 48 and $96 \mathrm{hr}$ after wounding, plus control mitotic rates, for a large circular initial wound. Figure 2(c) gives the mitotic rates at the same three times after wounding, plus control data, for a medium sized circular initial wound. More experimental details concerning Fig. 2(b) and 2(c) can be found in Sandvig et al. (1994).

\subsection{MODELLING MITOSIS: INSIGHT FROM PREVIOUS MODELS}

We define the mitotic rate in a manner that corresponds to making the above experimental 
measurements on the modelling data. It takes the form

mitotic rate

for region $\mathfrak{R} @$ time $t=$

$$
\begin{gathered}
\left(\int_{\mathfrak{R}} \mathrm{d} A^{\prime} \text { Total Cell Density }\left(A^{\prime}, t\right)\right. \\
\times \int_{\mathfrak{R}} \mathrm{d} A^{\prime} \int_{t-4 \mathrm{hr}}^{t} \mathrm{~d} t^{\prime} \mathfrak{M}\left(A^{\prime}, t^{\prime}\right)
\end{gathered}
$$

where $\mathrm{d} A^{\prime}$ is a measure of area (2-D), or length (1-D), and $\mathfrak{M} \delta A \delta t$ is the number of cell divisions occurring in the time interval $[t, t+\delta t]$ and in the region of space associated with $\delta A$. $\mathfrak{R}$ is the region over which the experimental measurements took place, i.e. the spatial region in which, for a given plot on the above experimental graphs, one counted the number of cells stopped in mitotic metaphase. For the experimental results above there are 16 such regions, centred on the marks on the horizontal axes of the above graphs, which correspond to each measurement made.

One may consider predictions of the above mitotic rate made by the previous model of Dale, Sherratt and Maini (1994a, b), hereby referred to as the DSM model. This study was mainly based in cartesian coordinates* and was one-dimensional. We briefly recap the form of this model. Using $m(x, t)$ to denote the cell density with $c(x, t)$ the concentration of chemical stimulus, the DSM model on a finite domain is the following set of coupled nonlinear partial differential equations

$$
\begin{gathered}
\frac{\partial m}{\partial t}=\frac{\partial}{\partial x}\left((\alpha+\bar{\alpha} c) \frac{\partial m}{\partial x}\right) \\
+(\rho+\bar{\rho} c)\left(m-\lambda m^{2}\right)-k m \\
\frac{\partial c}{\partial t}=D_{c} \frac{\partial^{2} c}{\partial x^{2}}+f(m)-h(m) c-\delta c,
\end{gathered}
$$

*As the geometry of eyeball curvature did not greatly affect the predictions of the model (Dale et al., 1994b; Gaffney et al., 1997). on a finite domain, with zero flux boundary conditions. The parameters, $\alpha, \bar{\alpha}, \rho, \bar{\rho}, \lambda, k, D_{c}$ and $\delta$ are constant, and $x$ denotes the cartesian spatial coordinate measuring distance along the basal lamina. The kinetics of both $m$ and $c$ are both of the form "Production-Loss". The function $f(m)$ represents a cell dependent source of stimulus, while $-h(m) c$ models the cell internalisation of stimulus. Further details concerning parameters, parameter estimation, motivations and the most appropriate functional forms of $f(m)$ and $h(c)$ may be found in the original papers (Dale et al., 1994b; Gaffney et al., 1997). As $x$ is a cartesian spatial coordinate,

(1) reduces for the DSM model to mitotic rate $(x, t)=$

$$
\begin{gathered}
{\left[\int_{x_{\text {low }}(x)}^{x_{\text {high }}(x)} \mathrm{d} x^{\prime} \text { Total Cell Density }\left(x^{\prime}, t\right)\right.} \\
\times \int_{x_{\text {low }}(x)}^{x_{\text {high }}(x)} \mathrm{d} x^{\prime} \int_{t-4 \mathrm{hr}}^{t} \mathrm{~d} t^{\prime} \mathfrak{M}\left(x^{\prime}, t^{\prime}\right) \\
x_{\text {low }}(x)=\max \left(x_{\min }, x-\Delta\right) \\
x_{\text {high }}(x)=\min \left(x_{\max }, x+\Delta\right) \\
\text { Time }=24 \text { hours: mitotic rate (\%) pred } \\
\text { by the Dale-Sherratt-Maini model }
\end{gathered}
$$

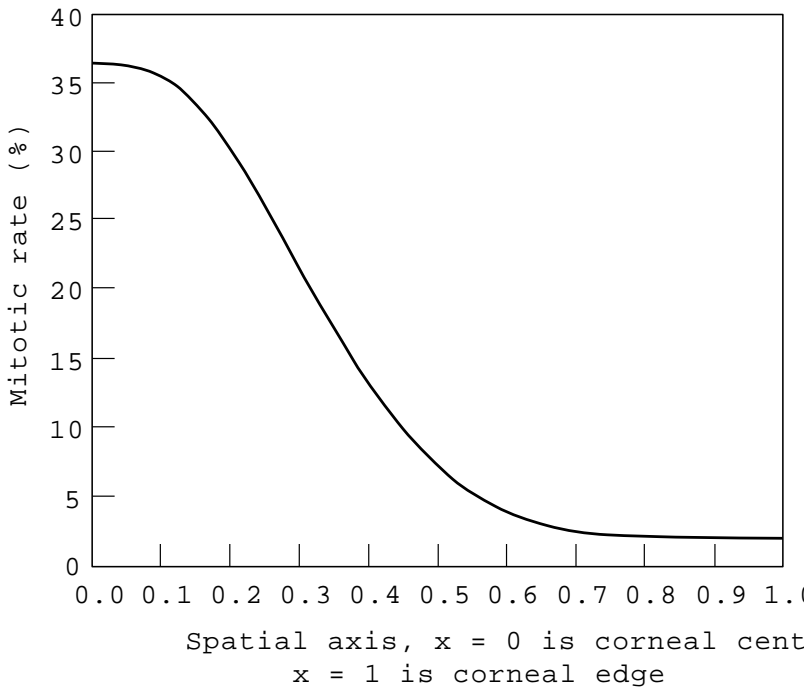

FIG. 3. Predictions from the DSM model for the mitotic rate across the cornea 24 hours after wounding. The plot is for half a cornea, with the corneal centre at $x=0$ and the limbal edge at $x=1$. Note the dramatic rise of predicted mitotic rate into the centre of the cornea, in contrast to the trends of the experimental data in Fig. 2. 
where $x_{\min }, x_{\max }$ denote the upper and lower limits of the one-dimensional spatial domain, and $\Delta$ is chosen so that, away from the boundaries, $(x-\Delta, x+\Delta)$ corresponds to $(1 / 16)$ th of the cornea. Hence $\Delta=(1 / 32) \times\left(x_{\max }-x_{\min }\right)$, and the averaging occurs over the same scale as in the experimental observations, where measurements were taken in 16 regions of the cornea.

The above mitosis function (2) for the DSM model for 24 hours after a small central slit wound yields the results shown in Fig. 3 (parameters as in the first model studied in Dale et al., 1994b).

Figure 3 is typical of all the mitotic rate predictions of the DSM model, for any parameter regime tested. Thus we see that the previous modelling framework predicts that the mitosis function increases rapidly into the corneal centre, which is clearly inconsistent with the experimental data.

The DSM model fails to capture the basis trend of the experimentally measured mitotic rates in Fig. 2, which always decrease into the wound. This naturally leads to the question of whether the trends exhibited by the experimental data may be captured by the use of more detailed, but still relatively simple, modelling. Investigating such a possibility is the overall aim of this paper. Firstly, one must consider why the DSM model fails in its predictions. Its main fault, for cell kinetic predictions, is that its formulation does not distinguish between proliferative cells and quiescent cells. Hence, all cells have the same proliferative ability, including those at the wound edge, and those at the centre of a recently covered wound bed. This leads to the erroneous predictions shown in Fig. 3, especially in regions where the cell density is small, such as a freshly covered wound bed, due to a low value of the denominator term in eqn (2). The fundamental difference that pervades the work presented in this paper is the introduction of two cell compartments: proliferative cells and quiescent cells. The proliferative cells constitute cells capable of undergoing a further round of mitosis, such as transient amplifying cells in the corneal epithelium. The quiescent cells are post-mitotic and thus represent the surface and (most of) the wing cell layers of the corneal epithelium.
By considering a model framework with two such compartments one may have sufficient structure to avoid the problems associated with the mitotic rate predictions of the DSM model. There is little or no proliferation at the wound edge (Crosson et al., 1986) during all stages of the wound healing, and so one may consider how such a situation arises in a two cell compartment model framework. One might speculate that the proliferative cells are present at the wound edge, but their proliferative capabilities are suppressed. However, this would be impossible to reconcile with the notion, based on experiment (Dunn \& Ireland, 1984; Barrandon \& Green, 1987), that the wound bed acts as a source of stimulus, such as growth factors, which stimulate mitosis as well as migration. Consequently, one is led to consider mechanisms whereby the proliferative cells are poorly represented at the wound edge.

There are two straightforward possibilities: heterogeneity of the proliferative cell distribution and differential migration rates of the proliferative and quiescent compartments. Heterogeneity of the proliferative cell distribution is commonly observed (Ebato et al., 1987, 1988; Tseng, 1989). To motivate the possibility of differential migration rates, we need to consider cell movement mechanisms. Although a number of details of such mechanisms are currently unknown, it is generally agreed that movement will increase the hydrostatic pressures within the cell (Bray, 1992). However, increased hydrostatic pressure would inhibit spindle formation in mitosis (Wolfe, 1995), thus suppressing proliferation. Consequently, one may consistently speculate that the intercellular conditions required for cell movement and proliferation are not compatible, which would result in differential migration rates for the proliferative and quiescent cell compartments.

The first model, denoted Model I below, incorporates simply differential migration rates, and we find this inadequate in predicting the observed cell kinetics. We are thus led to a second model, denoted Model II below, where we additionally consider the spatial heterogeneity of the corneal epithelium, motivated from the observation that stem cells, the ultimate progenitors of the corneal mass, are localised in 
the limbus (Tseng, 1989). We find that even these two mechanisms combined are not capable of reproducing some of the important trends exhibited by the experimental data. The manner in which these two models fail to reproduce the experimental data motivates the inclusion of a representation of juxtacrine signal in the third and final model of this paper, denoted Model III below. This model yields substantially better agreement between the mitotic rate predictions and the experimental data.

\section{Modelling Framework}

The modelling framework presented here encompasses the basic assumptions concerning the generic high level features of all models considered in this paper. As commented above, this represents a generalisation of the DSM model which includes distinct proliferative and quiescent cell compartments. Detailed assumptions have to be made in order to implement the model, which differ between given realisations of the general model framework; three of these are developed in later sections. The constituents of the model are:

- two generic epithelial cell types, together with a single stimulus, representing the net effects of growth factors and other possible stimuli.

As discussed above, one cell type is considered to be a proliferative (P) cell type, with the other a quiescent $(\mathrm{Q})$ cell type. The modelling of two distinct cell types is the key structure that is radically different from the previous modelling of CEWH. This enables modelling of both the heterogeneity of the proliferative cell compartment and the possibility that proliferative cells and quiescent cells migrate at different rates. The model obviously must simulate how its constituents migrate during CEWH.

- The chemical stimulus is assumed to spread rapidly across the cornea via a constant rate of transport, which will be modelled by a diffusion process.

- Both cell types are assumed to migrate as though they were diffusing substances. Cell migration is much slower than chemical stimulus transport, with the $\mathrm{P}$ cells migrating at a significantly slower rate than the Q cells. The cell migration rates increase significantly with increasing concentrations of the chemical stimulus.

The model also requires rules governing how the various constituents interact with each other, and how they are "created and/or destroyed" during CEWH. The general assumptions behind the rules in all the models considered are as follows:

- the proliferative (P) cells can undergo mitosis, which is enhanced by increases in the chemical stimulus;

- the $\mathrm{P}$ cells can also differentiate into quiescent (Q) cells. The differentiation is also enhanced by increases in the chemical stimulus;

- at small $\mathrm{P}$ cell densities the mitosis dominates, whereas at large $\mathrm{P}$ cell densities the differentiation dominates;

- cell desquamation, i.e. cell loss via the shedding of surface cells into the tear film, is represented by an exponential decay of $\mathrm{Q}$ cells with a half-life consistent with the estimates of the rate of corneal epithelial cell shedding. It should be noted that such estimates do vary significantly between different experimental groups (see Appendix A.3);

- a source of P cells at the limbus is included in the model to represent the stem cell production of transient amplifying cells in this region. This source is modelled as dependent on the chemical concentration;

- the chemical stimulus is assumed to decay exponentially, with a half-life the order of the half life of epidermal growth factor;

- the cells are assumed to internalise the chemical-receptor complex formed on the cell surface, which acts effectively as a cell-dependent chemical stimulus degradation process;

- additional sources of the chemical stimulus are often assumed to be present, as detailed below. 
3.1. QUALITATIVE FORM OF THE GENERAL MODEL FRAMEWORK

A qualitative summary of the above discussion is given by

Rate of change $=$ Chemically stimulated + of $\mathrm{P}$ cell density $\mathrm{P}$ cell diffusion where $H(r)=\operatorname{Heaviside}\left(r^{l m b l}-r\right) ; \quad r^{l m b l}=$ Position of inner edge of limbus, and $\nabla$ represents the appropriate vector calculus operator in terms of $r$. Throughout this paper, we use cylindrical
Rate of change $=$ Chemically stimulated $-\mathrm{Q}$ Cell of $\mathrm{Q}$ cell density $\mathrm{Q}$ cell diffusion

Rate of change $=$ chemical diffusion of chemical conc via tear film desquamation

+ Production
Chemically stimulated $\mathrm{P}$ cell differentiation

+ Chemically stimulated limbal source $\mathrm{P}$ cell production

+ Newly differentiated cells

- Decay

Removal by
$\mathrm{P}$ and $\mathrm{Q}$ cells where the terms "Chemically stimulated $\mathrm{P}$ cell differentiation" and "Newly differentiated cells" are taken to be equal in the above.

\subsection{EQUATIONS FOR GENERAL FRAMEWORK}

The form of the equations representing the above ideas is as follows. First of all, we list independent and dependent variables

$P$ : proliferative cell density

$Q$ : quiescent cell density

$c$ : stimulus concentration

$t$ : time

$r$ : cylindrical polar coordinate; corneal centre at $r=0$, outer limbal edge at $r=R$.

The equations, in their most general form, are

$$
\begin{gathered}
\frac{\partial P}{\partial t}=\nabla \cdot\left(D_{2}(c) \nabla P\right)+M(c, P ; \text { stem; } t, r) P \\
-F(c, P ; \text { stem; } t, r) \frac{P^{2}}{P^{\prime}}+\varsigma(c) H(r) \\
\frac{\partial Q}{\partial t}=\nabla \cdot\left(D_{1}(c) \nabla Q\right)-\sigma Q+F(c, P ; t, r) \frac{P^{2}}{P^{\prime}} \\
\frac{\partial c}{\partial t}=D_{s t i m} \nabla^{2} c+A+L(c, P+Q)-\delta c
\end{gathered}
$$

*This interpretation for $P^{\prime}$ will change slightly, though in a clear manner, for the later models. polar coordinates. One might instead choose to represent the vector calculus operators in terms of the angles of spherical, or even ellipsoidal, polar coordinates to better reflect eyeball curvature in the model. However, the discussion in Appendix B indicates that this will not change the results significantly.

The constant $A$ represents a constant tear film source of chemical stimulus as used and motivated in the DSM model (Dale et al., 1994a, b). The functions $D_{2}(c)$ and $D_{1}(c)$ control the diffusion of the proliferative and the quiescent cells, and hence from the above discussion one has $D_{1}(c)>D_{2}(c)$. The term $\varsigma(c) H(r)$ represents a source of proliferative cells at the limbal edge of the cornea, thus portraying the role of the stem cells.

The function $M(c, P$; stem; $t, r) P$ represents mitosis in any given model realisation, while $F(c, P$; stem; $t, r)\left(P^{2} / P^{\prime}\right)$ represents the differentiation of proliferative cells into quiescent cells. It is convenient not to absorb the parameter $P^{\prime}$ into the function $F(c, P$; stem; $t, r)$; for Model I $P^{\prime}$ has a natural interpretation of being, to a very good approximation, the equilibrium proliferative cell density in the cornea centre*, as motivated below. In the simple model initially presented the functions $M(c, P$; stem; $t, r) P$ and $F(c, P$; stem; $t, r)$ are taken to depend on $c$ and $P$. In the most general case, however, these 
functions might be complex, and could plausibly be argued to explicitly involve the stem cell activity, i.e. the value of $\varsigma(c) H(r)$ or even the history of the stem cell activity. Hence the argument "stem" for $M$ and $F$ in the framework equations above. We return to this below where we discuss a possible, though admittedly crude, caricature of such complex dependence. The accompanying boundary and initial conditions for the above equations are

$$
\begin{gathered}
\frac{\partial P}{\partial r}=\frac{\partial Q}{\partial r}=\frac{\partial c}{\partial r}=0 \text { at } r=0 \text { and } r=R \\
P(r, t=0)=\left\{\begin{array}{cc}
0 & r \in \text { Initial Wound } \\
P^{e q m}(r) & r \notin \text { Initial Wound }
\end{array}\right. \\
Q(r, t=0)=\left\{\begin{array}{cc}
0 & r \in \text { Initial Wound } \\
Q^{e q m}(r) & r \notin \text { Initial Wound }
\end{array}\right. \\
c(r, t=0)=\left\{\begin{array}{cc}
0 & r \in \text { Initial Wound } \\
c^{e q m}(r) & r \notin \text { Initial Wound }
\end{array}\right.
\end{gathered}
$$

where $P^{\text {eqm }}, Q^{\text {eqm }}$, and $c^{\text {eqm }}$ are the steady-state solutions of (3) for the above boundary conditions. The zero flux boundary conditions at $r=0$ are required for the Laplacians to be well defined there. The zero flux boundary conditions for the cell densities at the limbal edge are so that the model does not incorporate effects due to "transdifferentiation at the limbus", which have been virtually ruled out experimentally (Tseng, 1989).

For the chemical concentration, the zero flux boundary condition at the limbal edge is justified by the fact we have already included a simple caricature of the tear fluid source of chemical, via the term $A$ above. Including an extra source at the edge of the limbus would essentially represent a double counting of chemical sources. As we are using axisymmetric cylindrical polars, the equation for the mitosis function (1) reduces to

mitotic rate $(r, t)=$

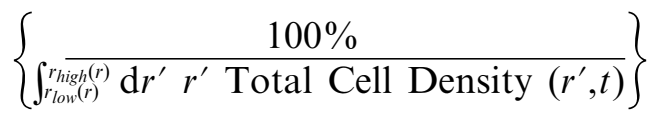

$$
\begin{gathered}
\times \int_{r_{\text {low }}(r)}^{r_{\text {high }}(r)} \mathrm{d} r^{\prime} r^{\prime} \int_{t-4 \mathrm{hr}}^{t} \mathrm{~d} t^{\prime} \mathfrak{M}\left(r^{\prime}, t^{\prime}\right) \\
r_{\text {low }}(r)=\max (0, r-\Delta) \\
r_{\text {high }}(r)=\min (R, r+\Delta),
\end{gathered}
$$

where $r$ is the radial coordinate and $R$ corresponds to the limbal edge of the cornea. The parameter $\Delta$ is chosen so that, away from the boundaries of the domain, $(r-\Delta, r+\Delta)$ corresponds to $(1 / 16)$ th of the radial length scale of the cornea, and so $\Delta=(1 / 32) R$. In terms of the model framework, the mitosis function is thus given by setting

$$
\begin{aligned}
& \text { Total Cell Density } \equiv(P+Q) \\
& \mathfrak{M} \equiv M\left(c, \mathrm{P} ; \text { stem; } t^{\prime}, r\right) P\left(r, t^{\prime}\right)
\end{aligned}
$$

in eqn (6).

\section{A Simple Model Realisation: Model I}

The simplest realisation of the above framework is now developed. The details required to implement this model are as follows:

- the diffusion rate of the $\mathrm{P}$ cells is proportional to that of the Q cells, and both are taken to be of the simple form

$$
D_{2}(c)=\kappa D_{1}(c)=\kappa\left(\alpha+\bar{\alpha} c^{n}\right) \quad \text { with } \quad 0<\kappa<1
$$

where $\alpha, \bar{\alpha}$ are constants and $n$ is a constant which is used to represent the nature of the response in the cells' activities to the presence of stimulus;

- the rate of mitosis $M(c, P$; stem; $t, r)$ and the rate of differentiation $F(c, P$; stem; $t, r)$ are assumed to be autonomous and homogeneous, with no stem cell dependence.

We use the simplifying assumption that $M(c, P$; stem; $t, r)$ and $F(c, P$; stem; $t, r)$ have the same type of dependence on the chemical stimulus, which would be consistent with the idea that the chemical stimulus decreases the cell cycle time and has also been used in previous models (Dale et al., 1994a, b). Specifically we 
take the function describing mitosis and differentiation to be

$$
\begin{aligned}
M(c, P ; \text { stem; } t, r) & =M(c, P)=\left(\rho+\bar{\rho} c^{n}\right), \\
F(c, P ; \text { stem; } t, r) & =F(c, P)=\left(\rho+\bar{\rho} c^{n}\right) .
\end{aligned}
$$

Implicit in these expressions is the assumption that one does not reach the stage where the effect of the chemical saturates, as would happen in reality for extremely large levels of stimulus. At equilibrium, the proliferative cell mitosis and differentiation at the limbus takes the form

$$
\frac{1}{T}\left(P-\frac{P^{2}}{P^{\prime}}\right)
$$

- The source term of proliferative cells is assumed to be

$$
\varsigma(c)=\chi+\bar{\chi} c^{n}
$$

The model also requires a description of the processes which directly effect the production and degradation of the chemical stimulus. In addition to the natural decay, with a half-life determined by experimental data, and the constant source of chemical, represented by $A$, we need to represent the cellular internalisation and degradation of chemical stimulus. This takes the form of the term used and motivated in Dale et al. (1994a, b), i.e.

$$
\begin{gathered}
L(c, P+Q)=B(P+Q)-\frac{\mu c(P+Q)}{c+c} \\
B(P+Q)= \begin{cases}0 & P+Q>\left.0.8\left(P^{e q m}+Q^{\text {eqm }}\right)\right|_{r=0} \\
\psi & P+Q<\left.0.4\left(P^{\text {eqm }}+Q^{\text {eqm }}\right)\right|_{r=0} \\
\frac{\psi}{0.4}\left(0.8-\frac{(P+Q)}{\left.\left.\left(P^{\text {eqm }}+Q^{\text {eqm }}\right)\right|_{r=0}\right)}\right. & \text { Otherwise }\end{cases}
\end{gathered}
$$

where $T$ is defined to be the doubling time of proliferative cells at the limbus. Consideration of (8) and (9) yields

$$
\left(\rho+\bar{\rho}\left(c^{e q m}\left(r^{l m b l}\right)\right)^{n}\right)=1 / T,
$$

which simplifies the form of the non-dimensionalised equations.

We also briefly consider the equation for $P$ given in (3) under equilibrium conditions. Noting, a posteriori, that the equilibrium proliferative cell density is approximately constant in the central cornea [see Fig. 4(a) below], we see that these equations yield

$$
\begin{aligned}
\left.P\right|_{r=0} & \sim P^{\prime} \frac{M(c, P ; \text { stem } ; t, r=0)}{F(c, P ; \text { stem } ; t, r=0)} \\
& =\left.P^{\prime} \frac{M(c, P)}{F(c, P)}\right|_{r=0}=P^{\prime},
\end{aligned}
$$

and hence the natural interpretation of $P^{\prime}$ as the equilibrium proliferative cell density for the central cornea in Model I.
$B(P+Q)$ represents the effect of a wound bed source term. As discussed in Dale et al. (1994a, b), this term is motivated by experimental considerations (Dunn \& Ireland, 1984; Barrandon \& Green, 1987); it is zero when the cell density is at, or near, equilibrium values, and is high at low cell densities with a simple linear interpolation between such regimes. Substituting eqns (7)-(13) into eqns (3), together with the initial and boundary conditions $(4,5)$, yields Model I.

\subsection{NON-DIMENSIONALISATION AND PARAMETERS}

The non-dimensionalisation used for all models in this paper is described in detail in Appendix A, where the non-dimensionalised equations are given for Model I. The parameters for Model I and the other models are also given in this Appendix. Model simulation with the cells responding linearly to the stimulus does not appear promising as the upregulation of the mitotic response is then not sufficient to promite 

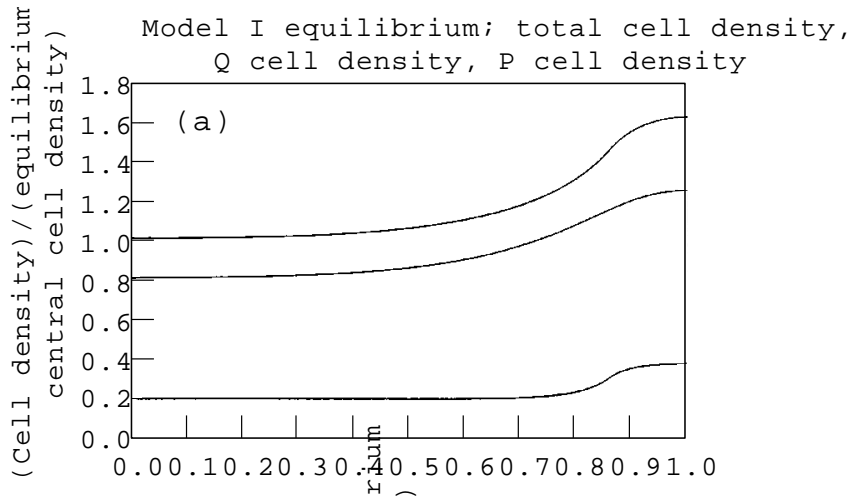

spatial axis, $r=\theta$ बिorneal centre, $r=1$ outer limbus

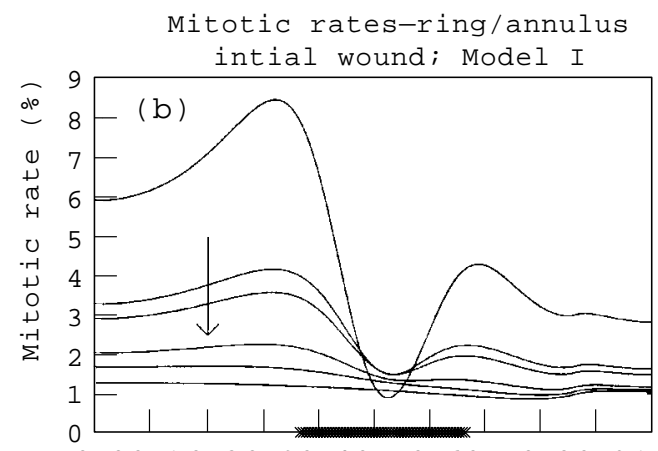

$0.00 .10 .20 .30 .40 .50 .60 .70 .80 .91 .0 \mathrm{~s}^{9}$

spatial axis, $r=0$ corneal centyre

$r=1$ outer limbus

Mitotic rates-large sized central wound; Model I

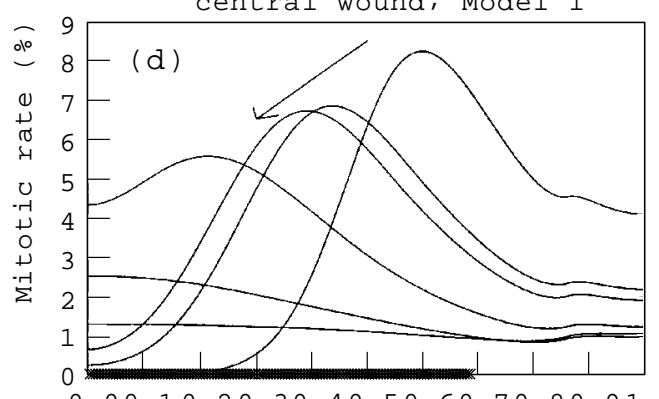

0.00 .10 .20 .30 .40 .50 .60 .70 .80 .91 .0

Spatial axis, $r=0$ corneal centyer

$r=1$ outer limbus

Mitotic rates-medium sized central wound; Model I

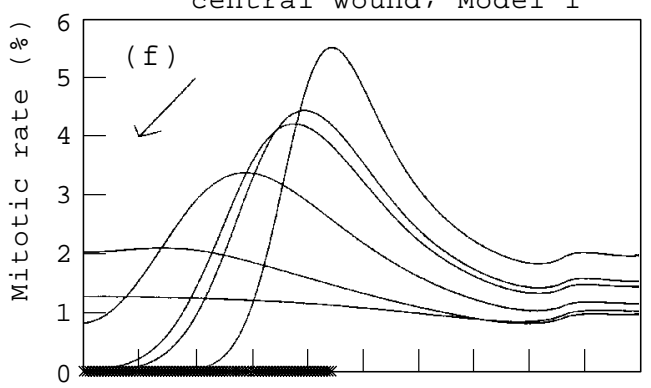

$0.00 .10 .20 .30 .40 .50 .60 .70 .80 .91 .0 \mathrm{G}$

spatial axis, $r=0$ corneal centre, $r=1$ outer limbus

(1)

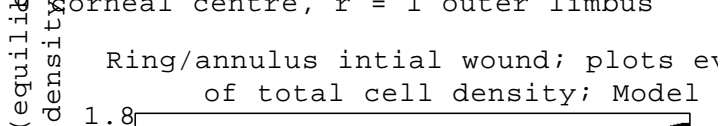
of total cell density; Model

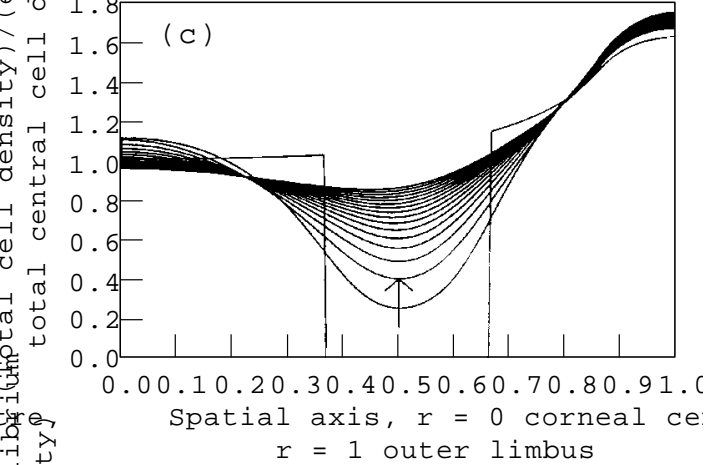

Large sized central wound; plots $6 \mathrm{hr}$ of total cell density; Mode

अ ⿷匚

$=-1.8-(e)$

त्र

U $1.4-$

न $1.2-$

H $1.0-$

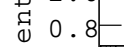

0

नु 0.4

$0.00 .10 .20 .30 .40 .50 .60 .70 .80 .91 . \mathrm{C}$

Spatial axis, $r=0$ corneal cel $r=1$ outer limbus

Medium sized central wound; plots 6 hr of total cell density; Mod $\epsilon$

$-1.6-(g)$

त्र

o $1.2-$

(

ช

-1,

O 0

0.6

0.4

0.

$$
\begin{gathered}
0.00 .10 .20 .30 .40 .50 .60 .70 .80 .91 . \mathrm{C} \\
\text { Spatial axis, } r=0 \text { corneal cel } \\
r=1 \text { outer limbus }
\end{gathered}
$$

FIG. 4. The upper plot, (a), is the Model I equilibrium prediction for P, Q, and total cell densities for the unwounded cornea, with the $\mathrm{P}$ cell prediction being of least magnitude; (b) is a plot of the mitotic rates for an initial ring wound, as indicated by the thick line on the horizontal axis. The times shown are 12, 24, 28, 48, and $96 \mathrm{hr}$ with time evolution indicated by the arrow. The lowest mitotic rate plot is the unwounded mitotic rate; (c) is the model prediction for the total cell density for the same initial wound, with plots every $6 \mathrm{hr}$, up until $96 \mathrm{hr}$. The analogues of the mitotic rate and cell density graphs for central large and central medium initial wounds are presented in (d)-(g). The type of initial wound is labelled on the mitotic rate graphs, as above, with mitotic plots at 12,24, 28, 48 and $96 \mathrm{hr}$ and cell density plots every $6 \mathrm{hr}$. Again, the unwounded mitotic rate is also plotted on the mitotic rate graphs and arrows indicate time evolution. The arrow indicates 
proliferation to the levels observed in the experimental data of Fig. 2. Hence the constant $n$, which determines the cells' sensitivity to the stimulus, is taken to be two. This is a common trend in the models of this paper, and $n$ is taken to be two for all the models presented. Indeed, it is well known that the response of cells to stimulus can often be nonlinear (Alberts et al., 1994).

It turns out that for Model I one has to take the parameters governing the stem cell production, namely $\chi$ and $\bar{\chi}$, to be unreasonably high in order to match experimental observation. This is because the stem cells drive the heterogeneity of this model. In turn, this entails that a high value for the stem cell production term is required to match the fact that there are approximately (10-12) cell layers in the limbus but only (5-6) cell layers in the central cornea (Dua et al., 1994). This requires the ratio of the cell densities between the limbus and the cornea to be between about 1.6 and 2.4. Throughout this paper we only consider parameter regimes which satisfy this condition, as it is a powerful, and well verified, constraint on any possible modelling. A stringent internal consistency condition that also must be satisfied is that the chemical and total cell densities at the corneal centre are unity, due to the choice of non-dimensionalisation.

The high stem cell levels in Model I also tend to drive other model parameters, such as desquamation rates, out of biologically reasonable bounds. Thus the first fault of Model I is that we are immediately driven to a non-biological parameter regime in order to match a concrete experimental observation. The parameter values used for Model I are therefore determined firstly by the requirement that the constraints discussed above are satisfied. The remaining parameters are chosen for consistency, to as great an extent as possible, within experimental bounds, which are discussed in the Appendix, and with the experimental data of Section 2.1. In fact, Model I robustly fails to reproduce the trends in this data. However, much insight can also be gained from observing

\footnotetext{
*i.e. a response corresponding to cell densities above that of the unwounded cornea.
}

in detail the ways in which the model is clearly inadequate. This can be seen from the typical trends exhibited by the results in Fig. 3, which are robust to parameter variation.

\subsection{DISCUSSION OF MODEL I RESULTS}

\subsubsection{Comparison with experimental data}

Figure 4 illustrates results from Model I, for parameters given in Appendix A.2. One can compare these predictions of the mitosis function with the experimental results in Fig. 2. The most striking aspect of the mitotic graphs predicted by Model I for the initial ring wound is the over-prediction, by orders of magnitude, of the mitotic levels in the central corneal epithelium for a ring-shaped initial wound. This can be seen by comparing the experimental data in Fig. 2(a) with the analogous graph from simulation results shown in Fig. 4(b). The model predictions for the mitotic rates following a large sized circular initial wound, Fig. 4(d), also do not capture the experimental data's trends at the limbus, as displayed in Fig. 2(b). The mitotic rates predicted after a medium sized circular initial wound, Fig. 4(f), are broadly similar to the experimental data, displayed in Fig. 2(c).

Note that the model prediction of the unwounded, equilibrium, mitotic rate in Fig. 4(b, $\mathrm{d}, \mathrm{f})$ is approximately constant, which is consistent with the control data presented in Fig. 2. Looking at the graphs for the predictions of the total cell densities, Fig. 4(c, e, g), we see that the model predicts a hyperplasic response* in the peripheral cornea in response to wounding, which is not observed in situ. This appears to be a common trend and occurs for all the model predictions in this paper, indicating that one is dealing with a complex system and thus cannot hope to capture all features of it with this level of modelling.

Another common trend throughout this paper for the total cell density plots is the lack of a well defined wound edge. This is due to the fact that the kinetics for $Q$ are not of "reaction" form, namely they are linear in $Q$ rather than a nonlinear function of $Q$ possessing node-saddle orbits in the phase plane, such as the function $Q(1-Q)$ characteristic of Fisher kinetics. This entails that the sharp transitions characteristic of 
reaction diffusion equations with Fisher kinetics do not occur. While this is a fault of the modelling, it could be easily rectified for a more sophisticated modelling effort, by use of a moving boundary formalism, which is well suited to CEWH modelling (Gaffney et al., 1998). It was decided not to implement such a scheme at this preliminary stage as the sharpness of the wound front is not responsible for the discrepancy between the predicted and measured mitotic rates in the ring wound.

\subsubsection{Model I conclusions}

The fact that mitotic rates for the initial annulus wound are severely overestimated is one of two failures of this simple model. This is not surprising; the combination of the wound bed source term together with, in the model, the presence of proliferative cells in the corneal centre makes this inevitable. The other, related, failing is that Model I relies on the observed heterogeneity of cell density in the cornea being induced solely by the presence of a limbal stem cell ring which leads to non-biological parameter regimes, as discussed in Section 4.1, above. However, the manner of these failings strongly indicate how the modelling should proceed.

The critical factor not captured by Model I is that central corneal cells are far less capable of undergoing proliferation than limbal cells, as has been confirmed by in vitro observations, where the proliferative abilities of cultures of these tissues differ (Danjo et al., 1987; Ebato et al., 1987)*. This common explanation for the heterogeneity in proliferative ability is an age structure of the proliferative cells, whereby the central cells are older and less capable of proliferation. Modelling age structure in detail, which would require rewriting the model for an age distribution $P(x, t$, age) (Hoppensteadt, 1975 ) is inappropriate given the sparsity of data available at the present time. However, the results from Model I clearly indicate that a caricature of age structure must be included.

\footnotetext{
* Note that these, and all other, references express such proliferative differences in terms of S-phase labelling indices, which cannot yield useful modelling information concerning cell doubling time, without additional data on the other phases of the cell cycle, especially the G-phases.
}

Model I can also be criticised in that if one were to model a low stem cell environment, i.e. with $\chi$ and $\bar{\chi}$ small or zero, it is still capable of supporting a non-trivial steady state of proliferative cells, yet in situ stem cells are well known to be critical to corneal stability. We thus proceed to develop Model II with the following aims in mind. We seek to produce a caricature model that does not support a non-trivial steady state of proliferative cells in the absence of stem cells, which corresponds to setting $\chi$ and $\bar{\chi}$ to zero in the model. We also want to investigate whether simple caricature modelling of age structure can improve the fit between model predictions and experimental data trends. We seek to gain insight into the likelihood of success of more detailed age structured modelling of the cell kinetics of CEWH should data become available to motivate such an approach. Further, we require a model that does not force biologically unreasonable parameters in order to reproduce experimental observations.

\section{Model II: A Caricature of Age Structure}

This model differs from Model $\mathrm{I}$, in that it contains a representation of age structure heterogeneity for the proliferative cells. It assumes that the ability of a given $\mathrm{P}$ cell to undergo mitosis varies, from high values near the limbus to low values near the corneal centre. One might also expect that a higher limbal stem cell source activity (over a sufficiently long period of time) will lead to a decrease of the average age of the $\mathrm{P}$ cells, and hence increased average rates of mitosis. Thus the mitotic rate in Model II is assumed to decrease as one moves away from the limbus towards the wound centre. It is also assumed to increase with increasing limbal source activity, but to saturate at high limbal source activity.

We change the mitosis function $M$, previously given by eqn (8), to the form

$$
\begin{aligned}
& M(c, P ; \text { stem } ;, r)= \\
& \quad\left(a+(1-a) \frac{r^{2}}{R^{2}}\right) \Lambda(\text { stem })\left(\rho+\bar{\rho} c^{n}\right) .
\end{aligned}
$$

We have normalised the term $(a+(1-a)$ $\left.r^{2} / R^{2}\right)$ to be unity at $r=R$, as required for 
eqn (10) to still hold, which implies that the interpretation of $T$ within Model II is the same as that for Model I. The term $\left(a+(1-a) r^{2} / R^{2}\right)$ crudely represents an age variation in the mitotic ability of the proliferative cells, via a spatial variation, on the premise that the younger proliferative cells tend to be situated in the limbal and peripheral cornea, since their source is the stem cells in the limbus. We tentatively take a quadratic variation as S-phase labelling data indicates (Ebato et al., 1987, 1988) that mitotic ability varies with $r$ nonlinearly with a power greater than one, though as mentioned above it is not possible to make a direct link between S-phase labelling data and doubling times without G-phase data. The parameter $a$ controls the level of heterogeneity, and the value used is given and discussed in Appendices A.2 and A.3.

The term $\Lambda$ (stem) is crude; it is meant to represent the variation of the proliferative ability with variation in the limbal source. For example, a decrease in the source activity will lead to an increase in the mean age of the proliferative cells, which, due to their capability to undergo a finite number of rounds of mitosis (Tseng, 1989), are thus less capable, on average, of undergoing mitosis. From this interpretation, we expect $\Lambda$ (stem) to tend to zero as the stem cell activity tends to zero, and consequently the model does not support a non-trivial steady state in the absence of stem cells (i.e. in the case where $\chi, \chi$ are set to zero). We also expect $\Lambda$ (stem) to be unity at equilibrium stem cell activity, and to increase and saturate if stem cell activity should increase higher than unwounded levels. We use the form

$$
\Lambda(\text { stem }) \equiv \vartheta\left(1-\left(1-\frac{1}{\vartheta}\right)^{\varsigma\left(c(R, t) / \varsigma^{h t h h(}\left(c^{e q m}(R)\right)\right.}\right)
$$

where $R$ is constant, referring to the edge of the limbus, and $\vartheta$ is the saturation level. The term $\varsigma^{\text {hlth }}\left(c^{\text {eqm }}(R)\right)$ is the equilibrium activity (for a healthy eye, with $\chi, \bar{\chi}$ fixed at levels associated with that of a healthy eye*). Here $\varsigma(c(R, t))$ is a crude representation of the stem cell activity at time $t$. Note that at equilibrium $\Lambda$ is unity as III.

*Which are also the values of $\chi, \bar{\chi}$ used in Models II and required. If there were no age structuring effects at all, $\vartheta$ would be unity, in which case $\Lambda$ would also be unity, as necessary for $\Lambda$ to have no effect on the cell kinetics. Also, we note that in the absence of stem cells, we have $\Lambda$ (stem) is zero, and thus no non-trivial stable state exists in the absence of stem cells for this model.

The above mitosis function (14), on the inclusion of (15), does not include a coupling between the spatial structure of the system and the history of the stem cell activity resulting in the omission of "time delay" effects. Hence, eqn (15) represents a crude caricature of the dependence of the changes of the proliferative ability with changes in the stem cell activity of the corneal epithelium. Systematic inclusion of such effects could only be possible within a detailed age structured model which, as mentioned, is inappropriate for the level of data available.

With this change in place, namely replacing (8) with (14), one can proceed with the non-dimensionalisation in exactly the way as Model I. This yields Model II.

Note that the interpretation of $P^{\prime}$ differs only slightly from Model I. We have, making the $a$ posteriori observation that from the equilibrium proliferative cell density is approximately constant at $r=0$ [see Fig. 5(a)], we have, analogously to Model I, that eqn (3), under equilibrium conditions, yields

$$
\left.P\right|_{r=0} \sim P^{\prime} \frac{M(c, P ; \text { stem } ; t, r=0)}{F(c, P ; \text { stem; } t, r=0)}=a P^{\prime},
$$

using the fact that $\Lambda$ (stem) is unity under equilibrium conditions. The only difference in the interpretation of $P^{\prime}$ from Model $\mathrm{I}$ is that now $a P^{\prime}$ yields the equilibrium central corneal profilerative cell density. Note that this interpretation of $a P^{\prime}$ also holds for Model III; we will discuss the further changes yielding Model III in Section 6.

\subsection{PARAMETER VALUES}

In contrast to Model $\mathrm{I}$, it is encouraging to note that it is possible to take parameters for Model II which result in predictions that satisfy the constraints of Section 4.1 and which are consistent with order of magnitude estimates based on experiment. This is detailed in Appendices A.2 and A.3. However, Model II 
also robustly fails to reproduce the trends of the experimental data in Fig. 2, as illustrated in Fig. 4. Again, the manner of this failure indicates how the modelling may be improved. The parameters used have been chosen to be consistent with the constraints of Section 4.1, and to satisfy the experimental bounds of the Appendix. By far the most important of any remaining freedom in the parameters is the limited freedom in the choice of $a$. This has been chosen to make the manner in which Model II robustly fails in the prediction of the trends exhibited by the data in Section 2.1 in all paramter regimes, as explicit as possible, as will be seen in the discussion below. As no new insight is obtained from examining the cell density plots of Model II we concentrate on the mitotic rate predictions of Model II.

\subsection{MODEL II DISCUSSION}

Figure 5 illustrates results from Model II, for parameters given in Appendix A.2. The first difference induced by the changes to the model can be seen by comparing the equilibrium plots of Models I and II, Figs 4(a) and 5(a), respectively. As expected for Model II, the P cell density decreases significantly into the corneal centre and is, on average, lower than for
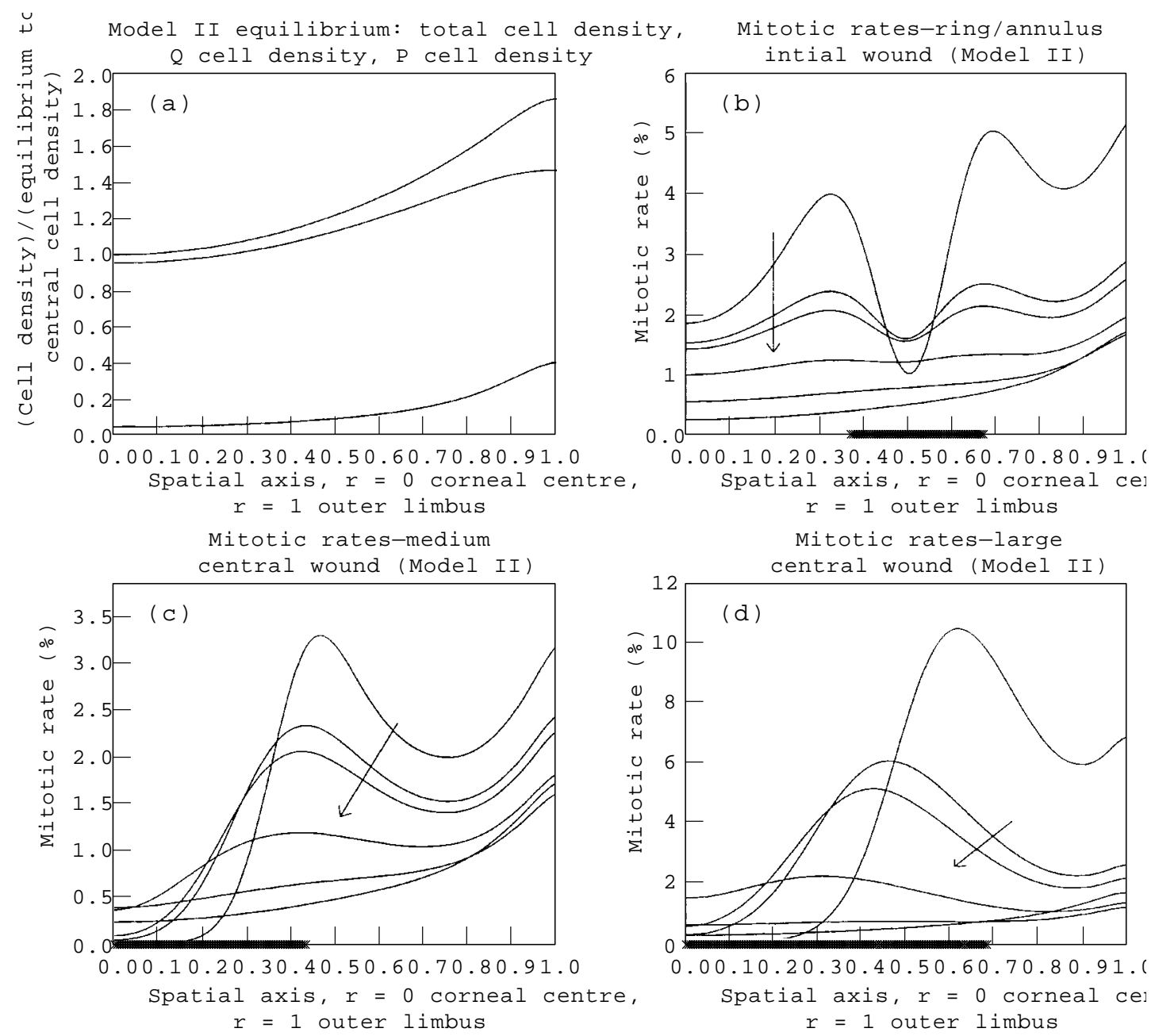

FIG. 5. The plot on the upper left above, (a), is the model II equilibrium prediction for P, Q, and total cell densities for the unwounded cornea. (Again, the $\mathrm{P}$ cell prediction is the one of least magnitude in the plot); (b) is a plot of the mitotic rates for an initial ring wound, as indicated by the thick line on the horizontal axis. The times shown are 12, 24, 28, 48 and $96 \mathrm{hr}$, where the time evolution is indicated by the arrow. The lowest mitotic rate on this graph is the unwounded mitotic rate; (c) given the model prediction for the mitotic rates for a medium central wound, while (d) gives the model prediction for the mitotic rates for a large central wound. The times shown are again 12, 24, 28, 48 and $96 \mathrm{hr}$ with an arrow indicating time evolution. The unwounded mitotic rate is also plotted on (b, c, d). Arrow indicates increasing time. 
Model I. By comparing Figs 4(b) and 5(b) we see that for a ring wound, Model II predicts a somewhat lower peak at the centre of the wound than Model I. It is still, however, an overprediction, being of the same magnitude as the peak in the corneal periphery, in contrast to the data of Fig. 2(a). For a large central initial wound, the limbal edge peak observed in the experimental data, Fig. 2(b), is still not captured by the model, by inspection of Fig. 5(d). In Fig. 5(c) we see that the Model II prediction for the mitotic rates after a medium sized initial wound still just, has a peak near the edge of the initial wound, but this is beginning to be dominated by a peak at the limbal edge, which arises due to the large number of $\mathrm{P}$ cells present at the edge compared with the edge of the initial wound. This limbal edge peak is not observed in the experimental data, Fig. 2(c). Further, the model predictions of the mitotic rates for medium and large central wounds in Fig. 5(c) and (d) indicate a sharp drop to zero mitosis as one approaches the corneal epithelial centre, which is not observed in the experimental data, Fig. 2(b), (c). The model predictions of the unwounded, equilibrium, mitotic rate only tenuously agree with the controls presented by the experimental results.

\subsection{MODEL II CONCLUSION}

In summary, Model II is a significant improvement on Model I. It satisfies the internal consistency conditions of Section 4.1 for biologically reasonable parameters. It also has reduced the extent of the greatest deviation of Model I from the observed experimental data, namely the mitotic rates in the central region of a ring wound. However, it still is not satisfactory in its ability to capture the trends of the experimental data of Fig. 2.

The results presented above illustrate the manner in which Model II is unsatisfactory. Recall that variations of $a$ in eqn (14) control the affects of age structure in Model II. Such variations can significantly change the results and reduce the overprediction in the central corneal mitotic rate for a ring wound, but at the expense of eliminating the peak of the mitotic rates for a medium sized wound. One may deduce from the results above that there is not a "window" in the possible values of $a$ for which the central mitotic rates for the ring wound are not overpredicted and for which the peak of the mitotic rates for medium sized wounds is not eliminated. This clearly indicates that the crude age structuring as developed in Model II is not sufficient to produce a model within the simple framework presented which does not contradict the trends illustrated in the experimental data.

Consequently, one is led to consider some additional mechanism to produce a model more capable of reproducing the trends observed by the experimental studies. It is important to note that any mechanism which affects the proliferative ability of the cells across the corneal epithelium in a uniform manner is unlikely to produce better results than we have seen for Model II, as it will probably suppress the central peak of the ring wound only at the expense of the presence of a mitotic peak in the medium wound. Consequently, the results from Model II encourage us to consider a mechanism which exhibits some form of "threshold" behaviour. This is addressed in our final model in which a biologically plausible extra mechanism is hypothesised. This is shown to produce results with trends that match the experimental ones, except for a discrepancy in the limbal region of the large central corneal wound.

\section{Model III}

As we have seen, even heterogeneity induced by a caricature age structuring is not sufficient to stop Model II from substantially overpredicting mitosis in the corneal centre for a ring wound.

We wish to keep to the aims stated at the end of Section 4.2.2 and, in addition, consider a biologically plausible extension to the model that uses the insight gained from Model II. We thus proceed to amend the existing model by assuming that cell mitosis and differentiation is controlled not only by a diffusible regulator, but also by a nearest-neighbour (juxtacrine) mechanism. This would correspond to signalling via one of the many growth factors that exist primarily in a membrane bound form [see Masssagué \& Pandiella (1990) for review of juxtacrine signalling]. There is no question that there are many different chemical stimuli involved in corneal epithelial wound healing (Jain \& Azar, 
1994; Gipson \& Inatomi, 1995). To our knowledge, there is no direct evidence for juxtacrine signal within the corneal epithelium, but its existence is highly plausible, and such a mechanism is known to play a role in wound healing of the epidermis (Stascheck et al., 1992; Schultz et al., 1987). Note that such a mechanism exhibits the threshold behaviour that is suggested from the results of Model II.

To be specific, we suppose that the diffusible signal primes the cells, with subsequent juxtacrine activation required to actually upregulate cell division, according to the presence of the chemical source. Implicit in our previous modelling, and previous models (Dale et al., 1994a, b), was the fact that the chemical stimulus affected the mitosis and differentiation functions in the same manner, which would be consistent with the idea that the chemical stimulus decreases the cell cycle time. We keep this implicit assumption, and thus consider the differentiation function, $F$, would be affected in a similar manner to the mitosis function $M$. This effectively leads to a threshold of $P$ cell stimulation, with a cutoff in chemically stimulated proliferation at the stage when the $P$ cells become so scarce that they are no longer connected to each other on the basal layer.

Thus for Model III, we redefine the mitosis and differentiation functions, $M$ and $F$ to be the central corneal epithelium (Forrester et al., 1996) a single cell layer corresponds to

$$
\left.P \sim 0.2\left(P^{e q m}+Q^{e q m}\right)\right|_{r=0},
$$

so this mechanism starts taking effect when less than one in three of the basal cells is a proliferative cell. The threshold level, $P^{0}$, corresponds to about one in five of the basal cells being proliferative; further details are discussed in Appendix A.3. For $P<P^{0}$, the chemical stimulus does not affect the cells. The sine function provides a convenient, monotonic and smooth interpolation for $G(P)$ between zero and unity when $P^{0}<\mathrm{P}<\mathrm{P}^{1}$.

\subsection{PARAMETERS}

The parameters used are given in Appendix A.2. We note that again it is possible to choose parameters for Model III which result in predictions that satisfy the constraints of Section 4.1 and which are also biologically reasonable, as detailed in Appendix A.3. The freedom left in the parameters after such consideration, as outlined in the Appendix, has been fixed by attempting to reproduce the trends of the experimental data in Fig. 2. Further details on parameters are given in Appendix A.3. Specifically, not that it is biologically reasonable parameter sets that yield the greatest similarity to the experimental observations. As an aside we note that,

$$
\begin{gathered}
M(c, P ; \text { stem; } t, r)=\left(a+(1-a) \frac{r^{2}}{R^{2}}\right) \Lambda(\text { stem })\left(\rho_{*}+\bar{\rho}_{*} G(P) c^{n}\right) \\
F(c, P ; \text { stem } ; t r)=\left(\rho_{*}+\bar{\rho}_{*} G(P) c^{n}\right) \\
G(P)= \begin{cases}0.0 & P \leqslant P^{0} \\
1.0 & P \geqslant P^{1} \\
0.5 *\left(1+\sin \left(\pi\left(2 P-P^{1}-P^{0}\right) /\left(2 *\left(P^{1}-P^{0}\right)\right)\right)\right. & P^{0}<P<P^{1}\end{cases}
\end{gathered}
$$

where

$$
P^{0}=\left.0.040\left(P^{e q m}+Q^{e q m}\right)\right|_{r=0} \quad P^{1}=\left.0.067\left(P^{e q m}+Q^{e q m}\right)\right|_{r=0} .
$$

We have introduced the function $G(P)$, which controls the effects of the juxtacrine mechanism. For $P>P^{1}$, there is no effect as $G(P)=1$. Note that as there are approximately five cell layers in concerning robustness, the trends of the model predictions do not appear to be sensitive to small variations in the parameters within the constraints of Section 4.1. 
Model III equilibrium: total cell density,

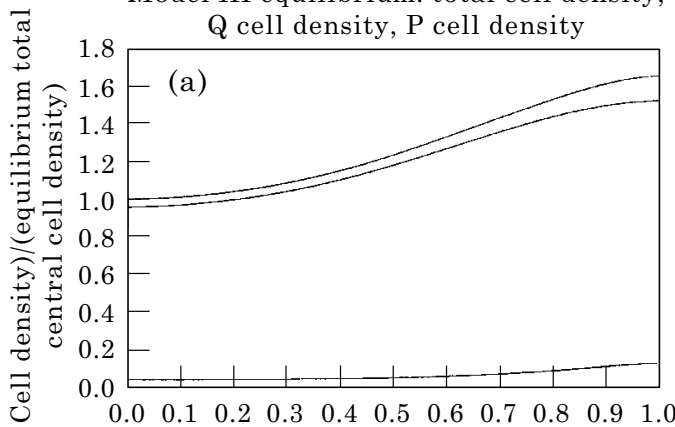

Spatial axis, $r=0$ central cornea, $r=1$ outer limbus
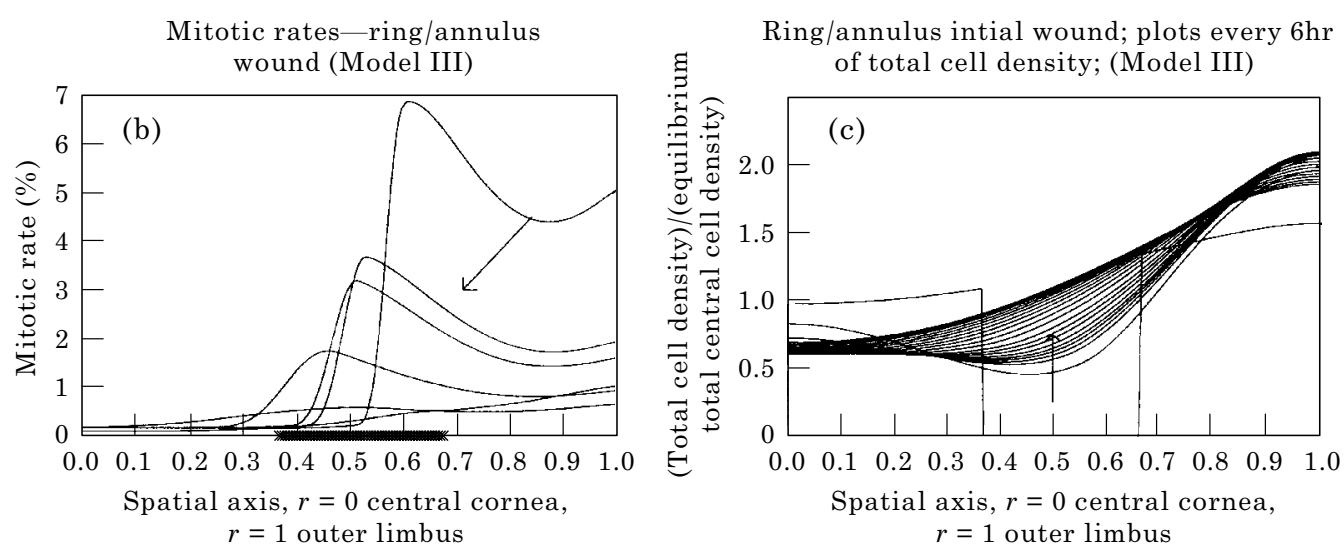

Mitotic rates-large central
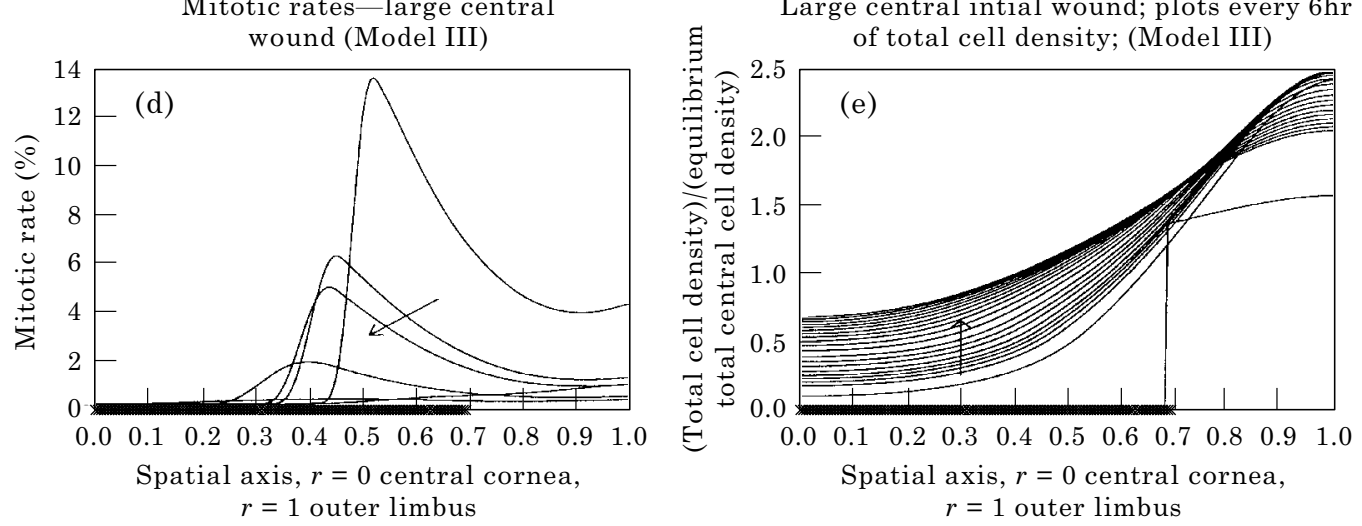

Mitotic rates-medium central wound (Model III)
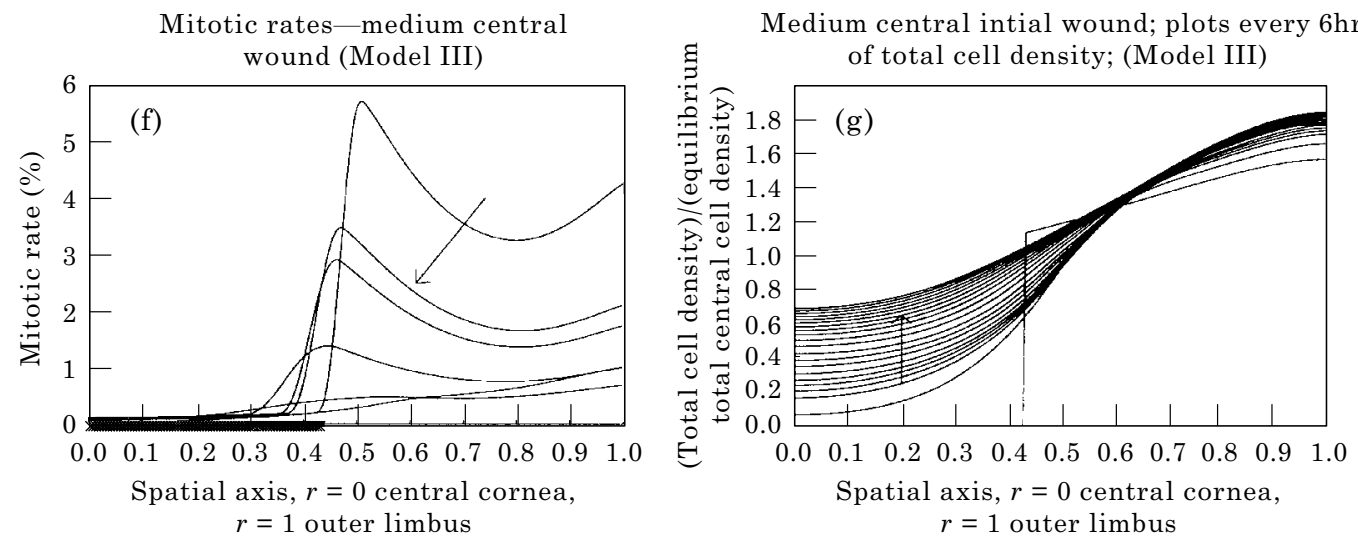

FIG. 6. The upper plot, (a), is the Model III equilibrium prediction for P, Q, and total cell densities for the unwounded cornea, with the $\mathrm{P}$ cell prediction being of least magnitude; (b) is a plot of the mitotic rates for an initial ring wound, as indicated by the thick line on the horizontal axis. The times shown are 12, 24, 28, 48, and 96 hr, where the time evolution is indicated by the arrow. The unwounded mitotic rate is also plotted, being the line nearest unity at $r=1$; (c) is the model prediction for the total cell density for the same initial wound, with plots every $6 \mathrm{hr}$, up until $96 \mathrm{hr}$. The analogues of the itotic rate and cell density graphs for central large and central medium initial wounds are given in (d, e) and (f, g), respectively. The type of initial wound is labelled on the mitotic rate graphs, as above, with mitotic plots at 12 , 24 , 28 , 48 and $96 \mathrm{hr}$ and cell density plots every $6 \mathrm{hr}$. Again the unwounded mitotic rate is also plotted on the mitotic rate graphs 
Thus substituting (17) into eqns (3), with the above parameters yields the dimensionalised model, which is non-dimensionalised as in Appendix A.

\subsection{MODEL III DISCUSSION}

Figure 6 illustrates the results from Model III, for parameters given in Appendix A.2; one can see that Model III is a substantial improvement on the earlier models. Its predictions given in Fig. 6(b) exhibit a general trend for the mitotic rates of the initial ring/annulus wound which is much closer to the trend exhibited in the experimental data in Fig. 2, without loss of the central peak in the medium wound mitotic rate prediction, depicted in Fig. 6(d). Hence, the predictions of Model III are broadly consistent with the predictions of the experimental data, except that there is an additional trough in the Model III mitotic rates in the limbal region of the cornea for the large central wound, Fig. 6(f), and a very small peak in the limbal region for a medium central wound, Fig. 6(d).

It should be noted from Fig. 6(b, d, f) that the unwounded, equilibrium, mitosis solutions, agree only tenuously with the experimental data, as with Model II; and that Model III suffers some problems common to all the models in the paper, concerning the prediction of hyperplasia, and the sharpness of wound fronts, as discussed in Section 4.2. The persistence of the latter problem concerning wound sharpness is understood to be an unavoidable consequence of the present modelling framework, as discussed in Section 4.2; the persistence of the former problem, concerning hyperplasia prediction, suggests that this too could also be an unavoidable consequence of the present modelling framework.

\section{Conclusions}

The initial motivating factor for the modelling investigation above is the development of a modelling framework capable of capturing cell kinetic data, such as that presented by Sandvig et al. (1993, 1994). This has required substantial extensions and improvements on previous modelling efforts. A key point is that modelling corneal epithelial wound healing $(\mathrm{CEWH})$ using spatially homogeneous kinetics is an over-sim- plification, as this cannot capture effects due to the stem cells present in the limbal ring acting as a source of proliferative cells. This source must be represented in any realistic modelling framework as it is of critical importance in CEWH.

Another key point is that the previous modelling efforts fail to capture a basic common trend of the data presented, namely that the model prediction of mitotic rates always decrease into the wound. This is because previous models do not distinguish between proliferative cells and quiescent cells in their formulation. Hence, all cells have the same proliferative ability, including those at the wound edge, and those at the centre of a freshly covered wound bed, leading to erroneous predictions as seen in Fig. 3.

The modelling framework presented above includes a proliferative and quiescent compartment, with the proliferative cells migrating at slower rates than the quiescent cells. This basic idea prevents model predictions of mitosis monotonically increasing as one moves into the central regions of the cornea. The results of such a modelling framework have been presented above. While the modelling framework realisations do not capture every trend of the experimental data for all given initial wound geometries, Model III captures many, by consideration of a juxtacrine mechanism. This represents a very substantial improvement in the field of modelling CEWH. One need only look at Fig. 3 to see the extent of this improvement. The fact that simple modelling can reproduce many of the trends of such a complicated system is, in itself, a substantial success.

This work also sets a foundation of understanding for further modelling investigation of the cell kinetics of CEWH. In particular it emphasises the importance of the coupling between spatial heterogeneity and age structure in the modelling of the corneal epithelium. It demonstrates that even caricatures of this complex process are not adequate to capture the trends exhibited by the experimental data. A simple juxtacrine mechanism is presented which yields results that mostly demonstrate peaks and troughs in mitotic activity in line with what one may 
expect from the experimental data, though there are additional caveats, as discussed above. Thus, we firstly see that further modelling work would require the inclusion of age structuring, but lack of experimental data prevents such an investigation at the present time. However, it should be noted that there is the promise of a great deal of new experimental insight and quantitative data from the application of confocal microscopy to the investigation of the cell kinetics and cell dynamics of corneal epithelial wound healing (Masters, 1995). Consequently one may be able to attempt such modelling in the foreseeable future, and the insights and results acquired in this paper would be extremely valuable.

In summary, the modelling predicts that the existence of proliferative and quiescent cell populations, age structuring of the populations and the existence of a juxtacrine signalling mechanism are all crucial to the observed pattern of corneal epithelial mitosis following wounding in the cornea.

We thank Tony Bron for helpful discussions. EAG is grateful for the financial support of a Wellcome Trust Postdoctoral Training Research fellowship. Part of this work has been supported by the London Mathematical Society, under scheme 3. This research (PKM) was supported in part by the Institute for Mathematics and its Application with funds provided by the National Science Foundation.

\section{REFERENCES}

Alberts, B., Bray, D., Lewis, J., RafF, M., Roberts, K. \& Watson, J. (1994). Molecular Biology of the Cell. New York: Garland Publishing.

Barrandon, Y. \& GreEn, H. (1987). Cell migration is essential for sustained growth of keratinocyte colonies: the roles of transforming growth factor- $\alpha$ and epidermal growth factor. Cell 50, 1131-1137.

Beebe, D. \& Masters, B. (1996). Cell lineage and the differentiation of corneal epithelial cells. Invest. Ophthalmol. Vis. Sci. 37, 1815.

Berg, O. \& Von Hippel, H. (1985). Diffusion controlled macromoecular interactions. Ann. Rev. Biophys. Chem. 14, 131

Bray, D. (1992). Cell Movements, pp. 149-150. New York: Garland Publishing.

Bron, A., Tripathi, R. \& Tripathi, B. (1997). Wolff's Anatomy of the Eye and Orbit. London: Chapman \& Hall.

Buck, R. C. (1985). Measurement of centripetal migration of normal corneal epithelial cells in the mouse. Investig. Ophthal. Vis. Sci. 26, 1296.

Chan, K. Y., Lindquaist, T., Edenfield, M., Nicholson, M. \& BANKs, A. (1991). Pharmacokinetic study of recombinant human epidermal growth factor in the anterior eye. Investig. Ophthal. Vis. Sci. 32, 3209.

Cotsarelis, G., Sun, T. \& Lavker, R. (1990). Label retaining cells reside in the bulge area of pilosebaceous unit: implications for follicular stem cells, hair cycle, and skin carcinogenesis. Cell 61, 1329-1337.

Crosson, C. E., Klyce, S.D. \& Beuerman, R. W. (1986). Ephithelial wound closure in rabbit cornea. Invest. Ophthalmol. Vis. Sci. 27, 464-473.

Dale, P. D., Maini, P. K. \& Sherratt, J. A. (1994a). Mathematical modelling corneal epithelial wound healing. Math. Biosci. 124, 127.

Dale, P. D., Sherratt, J. A. \& Maini, P. K. (1994b). On the speed of corneal epithelial wound healing. Appl. Math. Lett. 7, 11-14.

Danjo, S., Friend, J. \& Thoft, R. (1987). Conjunctival epithelium in healing of corneal epithelial wounds. Invest. Ophthalmol. Vis. Sci. 28, 1455.

DuA, H., Gomes, J. \& Singh, A. (1994). Corneal epithelial wound healing. Br. J. Ophthalmol. 78, 401.

Dunn, G. A. \& Ireland, G. W. (1984). New evidence that growth in $3 \mathrm{~T} 3$ cell cultures is a diffusion limited process. Nature 312, 63.

Ebato, B., Friend, J. \& Thoft, R. (1988). Comparison of limbal and peripheral human corneal epithelium in tissue culture culture. Invest. Ophthalmol. Vis. Sci. 29, 1533.

Ebato, B., Friend, J. \& Thoft, R. (1987). Comparison of central and peripheral human corneal epithelium in tissue culture culture. Invest. Ophthalmol. Vis. Sci. 28, 1450.

Forrester, J., Dick, A., McMenamin, P. \& Lee, W. (1996). The Eye. London: W. B. Saunders.

Gaffney, E. A., Maini, P. K., Sherratt, J. A. \& Dale, P. D. (1997). Wound healing in corneal epithelium: biological mechanisms and mathematical models. J. theor. Med. 1, 13-23.

Gaffney, E. A., Maini, P. K., McCaig, C., Zhao, M. \& Forrester, J. (1998). Modelling corneal epithelial wound closure in the presence of physiological electric fields via a moving boundary formalism (submitted).

Gipson, I. \& InATOMI, T. (1995). Extra-cellular matrix and growth factors in corneal wound healing. Curr. Opinions Ophthalmol. 6(IV), 3.

Hoppensteadt, F. (1975). Mathematical Theories of Populations: Demographics, Genetics and Epidemics. CBMS-NSF Regional Conference Series in Mathematics 20. Philadelphia: Society for Industrial and Applied Mathematics.

JAIN, S. \& AzAR, D. (1994). Extra-cellular matrix and growth factors in corneal wound healing. Curr. Opinions Ophthalmol. 5(IV), 3.

Klyce, S. D. \& Beuerman, R. (1988). Structure and function of the cornea. In: The Cornea, (Kaufman, H., Barron, B., Macdonald, M., Waltman, S., eds). New York: Churchill Livingstone.

KruSE, F. (1994). Stem cells and corneal epithelial regeneration Eye $\mathbf{8 ,} 170$.

Massagué, J. \& PANDiella, A. (1990). Membrane-anchored growth factors. Ann. Rev. Biochem. 62, 515-541.

Masters, B. (1995). Scanning slit confocal microscopy of the in vivo cornea. Opt. Engng 34, 684.

Ohashi, Y., Morokura, M., Kinoshita, Y., Mano, T., Watanabe, H., Kinoshita, S., Manabe, R., Oshiden, K. \& YANAIHARA, C. (1989). Presence of epidermal growth 
factor in human tears. Invest. Ophthalmol. Vis. Sci. 30, 1879.

ReI, H. \& Wilson, G. (1996). The cell shedding rate of the corneal epithelium - a comparison of collection methods. Curr. Eye Res. 15, 1054-1059.

SANDVIG, K. U. \& HAaskJOLD, E. (1993). The proliferative response during regeneration of a ring-shaped defect in the corneal epithelium. Acta Ophthalmol. 71, 39.

SANDVig, K. U., HAaskJold, E., BJERKnes, R., Refsum, S. B. \& KraviK, K. (1994). Cell kinetics of conjunctival and corneal epithelium during regeneration of different sized corneal epithelial defects. Acta Ophthalmol. 72, 43.

Schultz, G. S., White, M., Mitchell, R., Brown, G., Lynch, J., TwardziK, D. R. \& Todaro, D. J. (1987). Epithelial wound healing enhanced by transforming growth factor alpha and vaccinia growth factor. Science 235, 350-352.

Sharma, A. \& Coles, W. (1989). Kinetics of Corneal epithelial maintenance and graft loss. Invest. Ophthalmol. Vis. Sci. 30, 1962.

Stoscheck, C. M., Nanney, L. B. \& Lloyd, L. E. (1992). Quantitative determination of EGF-R during epidermal wound healing. J. Invest. Dermatol. 99, 645-649.

Tsai, L., Masters, B. \& BeEBe, D. (1997). Meeting Abstract: Coordination of mitosis and differentiation in the corneal epithelium. Invest. Ophthalmol. Vis. Sci. 38, 2281.

TSENG, S. (1989). Concept and application of limbal stem cells. Eye 3, 141.

Van Scott, E., Ekel, T. \& Auerbach, R. (1963). Determinants of rate and kinetics of cell division of scalp hair. $J$. Invest. Dermatol. 41, 269-273.

Wolfe, S. (1995). Introduction to Cell and Molecular Biology, p. 706. California: Wadsworth Publishing Company.

Wright, N. \& Alison, M. (1984). The Biology of Epithelial Cell Populations, Vol. 1. Oxford Science Publications.

Zieske, J., Bukosoglu, G. \& Yankauckas, M. (1992). Characterisation of a potential stem cell marker. Invest. Ophthalmol. Vis. Sci. 33, 143.

\section{APPENDIX A}

\section{Non-dimensionalisation \& Parameters}

In this Appendix non-dimensionalisation and parameters are discussed in detail. Firstly, we introduce the non-dimensionalisation scheme used in the modelling, and illustrate the non-dimensionalisation of Model I. The non-dimensionalisation of Models II and III proceed in an analogous manner. Parameter values for this non-dimensionalisation are presented, followed by a discussion of the experimental constraints on these parameters.

\section{A.1. NON-DIMENSIONALISATION}

We non-dimensionalise length scales with respect to the characteristic radius of the cornea, $R$. Time-scales are non-dimensionalised using a scale $T$, which is defined to be such that the average doubling time of proliferative cells at the limbus in the unwounded cornea is given by $\ln (2) T$, which leads to some simplification of the non-dimensionalised model. The non-dimensionalisation used is explicitly:

$$
\begin{aligned}
& r \rightarrow r_{*}=\frac{r}{R} \quad t \rightarrow t_{*}=\frac{t}{T} \\
& P \rightarrow P_{*}=\frac{P}{\left.\left(P^{e q m}+Q^{e q m}\right)\right|_{r=0}} \\
& \rho \rightarrow \rho_{*}=T \rho \quad c \rightarrow c_{*}=\frac{c}{c^{e q m}(r=0)} \\
& Q \rightarrow Q^{*}=\frac{Q}{\left.\left(P^{e q m}+Q^{e q m}\right)\right|_{r=0}} \\
& \alpha \rightarrow \alpha_{*}=\frac{\mathrm{T} \alpha}{\mathrm{R}^{2}} \quad \bar{\rho} \rightarrow \bar{\rho}_{*}=\left(\left.c^{e q m}\right|_{r=0}\right)^{n} T_{*} \bar{\rho} \\
& \bar{\alpha} \rightarrow \bar{\alpha}_{*}=\frac{\left(\left.c^{e q m}\right|_{r=0}\right)^{n} T \alpha}{R^{2}} \\
& \sigma \rightarrow \sigma_{*}=\frac{\sigma}{T} \quad D_{\text {stim }} \rightarrow D_{\text {stim* }}=\frac{T D_{\text {stim }}}{R^{2}} \\
& A \rightarrow A_{*}=\frac{T A}{\left.c^{e q m}\right|_{r=0}} \\
& c \rightarrow c=\frac{c}{\left.c^{e q m}\right|_{r=0}} \quad \delta \rightarrow \delta *=T \delta \\
& \mu_{*} \rightarrow \mu=\frac{\left.\mu_{*} T\left(P^{e q m}+Q^{e q m}\right)\right|_{r=0}}{\left.c^{e q m}\right|_{r=0}} \\
& r_{*}^{l m b l}=\frac{r^{l m b l}}{R} \\
& B(P+Q) \rightarrow B_{*}\left(P_{*}+Q_{*}\right)= \\
& T B\left(\left.\left(P_{*}+Q_{*}\right)\left(P^{e q m}+Q^{e q m}\right)\right|_{r=0}\right),
\end{aligned}
$$

Also, $\chi_{*}^{*}, \bar{\chi}_{*}, P_{*}^{\prime}$ are formed from a non-dimensionalisation analogous to that which yields $\alpha_{*}$, $\bar{\alpha}_{*}, \quad P_{*}$, respectively, while $k$ and $n$, being dimensionless constants, are unaffected.

Thus to non-dimensionalise Model I, for example, combine eqns (3), (5), (7)-(13), and (A.1) to yield

$$
\begin{aligned}
\frac{\partial P_{*}}{\partial t_{*}}= & \kappa \nabla *\left(\left(\alpha_{*}+\bar{\alpha}_{*} c_{*}^{n}\right) \nabla P_{*}\right)+M_{*}\left(c_{*}, P_{*} ; t_{*}, r_{*}\right) P_{*} \\
& -F_{*}\left(c_{*}, P_{*} ; t_{*}, r_{*}\right) \frac{P_{*}^{2}}{P_{*}^{\prime}}+\left(\chi_{*}+\bar{\chi}_{*} c_{*}^{n}\right) H\left(r_{*}\right)
\end{aligned}
$$




$$
\begin{gathered}
\frac{\partial Q_{*}}{\partial t_{*}}=\nabla_{*}\left(\left(\alpha_{*}+\bar{\alpha}_{*} c_{*}^{n}\right) \nabla Q_{*}\right)-\sigma_{*} Q_{*}+F_{*}\left(c_{*}, P_{*} ;\right. \\
\frac{\partial c_{*}}{\partial t_{*}}=D_{s t i m *} \nabla_{*}^{2} \mathrm{c}_{*}+A_{*}+B_{*}\left(P_{*}+Q_{*}\right) \\
-\frac{\mu_{*} \mathrm{c}_{*}\left(\mathrm{P}_{*}+\mathrm{Q}_{*}\right)}{\mathrm{c}_{*}+\mathrm{c}_{*}}-\delta c_{*} \\
H\left(r_{*}\right)=\operatorname{Heaviside}\left(r_{*}^{l m b l}-r_{*}\right)
\end{gathered}
$$

with the function $B_{*}$ given by

$B *\left(P_{*}+Q_{*}\right)$

$$
= \begin{cases}0 & P_{*}+Q_{*}>0.8 \\ \psi_{*} \frac{\left(0.8-\left(P_{*}+Q_{*}\right)\right)}{0.4} & 0.4<P_{*}+Q_{*}<0.8 \\ \psi_{*} & P_{*}+Q_{*}<0.4\end{cases}
$$

The functions $M, F$ are given by

$$
\begin{aligned}
& M_{*}\left(c_{*}, P_{*} ; t_{*}, r_{*}\right)=\left(\rho_{*}+\bar{\rho}_{*} c_{*}^{n}\right) \\
& \quad \text { with } M_{*}\left(1, P_{*} ; t_{*}, 1\right)=1 \\
& F_{*}\left(c_{*}, P_{*} ; t_{*}, r_{*}\right)=\left(\rho_{*}+\bar{\rho}_{*} c_{*}^{n}\right) \\
& \quad \text { with } F_{*}\left(1, P_{*} ; t_{*}, 1\right)=1
\end{aligned}
$$

where the constraints at $\left(c_{*}, r_{*}\right)=(1,1)$ arise from eqn (10) and are reflected in the parameter values given in the following section. These are accompanied by zero flux boundary conditions and initial conditions, which are the non-dimensionalised analogues of (5).

We proceed to list parameter values, some of which will depend on the form of the above non-dimensionalisation. It is important to note that differences in the non-dimensionalisation between this model and the DSM model entail that some parameter values inherited from the DSM model will take different numerical values.

\section{A.2. EXPERIMENTAL CONSTRAINTS ON ABOVE PARAMETERS}

Typically in biological systems precise parameter estimation is difficult or impossible, though one can often deduce the order of magnitude of various parameters from experimental data. A summary of such constraints is given in the section below. The motivation for the choice of parameters for each of the models is given in Sections 4.1, 5.1 and 6.1 for Models I, II, and III, respectively.

$R$ is based on the data and measurements of Sandvig et al. (1994). $r^{l m b l}$ has been taken from Sharma \& Coles (1989). The value of $c_{\text {eqm }}$ represents the equilibrium concentration of the chemical stimulus, which is taken to be of the order of the equilibrium, unwounded, concentration of EGF. This can be estimated via a study of reflex tears, which has been performed by Ohashi et al. (1989) who determined a value of about $7 \times 10^{-10} \mathrm{M}$, as used in Dale et al. $(1994 a, b)$. To the authors' knowledge, there is no detailed data for the cell cycle time of corneal transient amplifying cells. Experimental measurements of cell cycle times for varieties of epidermal transient amplifying cells range from 18-24 hr (Van Scott et al., 1963; Cotsareux, 1990). We consider these to be bounds for the $P$ cell cycle time, though the lower estimate of the cell cycle time yields values of mitosis in the model closer to that observed in the experimental data. Consequently, a value close to the lower bound is used in the models. The central cell density is estimated from the fact that a typical corneal epithelial cell length is about $10^{-5} \mathrm{~m}$ (Klyce \& Beuerman, 1988; Dale et al., 1994a, b). From non-dimensionalisation we have $\rho_{*}+$ $\bar{\rho}_{*}=1$.

One may produce a simple order of magnitude for the diffusion rate of cells in the unwounded cornea, which is (approximately) $\alpha_{*}+\bar{\alpha}_{*}$, as follows. Buck (1985) has measured the average equilibrium radial velocity of epithelial cells in the rabbit cornea to be

$$
V_{a v}=17 \mu \mathrm{m} \mathrm{day}^{-1} \sim 20 \mu \mathrm{m} \mathrm{day}^{-1}
$$

The total flux of cells in the model is given, for equilibrium conditions, by

$$
\begin{aligned}
\left(\alpha+\bar{\alpha}\left(\mathrm{c}^{\mathrm{eqm}}(\mathrm{r})\right)^{\mathrm{n}}\right)\left(\frac{\partial Q}{\partial r}+\kappa \frac{\partial P}{\partial r}\right) \\
\\
\sim(\alpha+\bar{\alpha})\left(\frac{\partial Q}{\partial r}+\kappa \frac{\partial P}{\partial r}\right)
\end{aligned}
$$


TABLE A.1 Parameters

\begin{tabular}{|c|c|c|c|c|}
\hline Parameter & Meaning & Model I & Model II & Model III \\
\hline$R$ & Radius of rat cornea & $0.4 \mathrm{~cm}$ & $0.4 \mathrm{~cm}$ & $0.4 \mathrm{~cm}$ \\
\hline$r^{l m b l}$ & Normalised limbus & $0.86 R_{*}$ & $0.86 R_{*}$ & $0.86 R_{*}$ \\
\hline $\ln (2) T$ & $\begin{array}{l}\text { Average } \mathrm{P} \text { cell } \\
\text { cycle time }\end{array}$ & 1 day & 1 day & 1 day \\
\hline$\left.\left(P^{e q m}+Q^{e q m}\right)\right|_{r=0}$ & Central cell density & $10^{15}$ cells $\mathrm{m}^{-3}$ & $10^{15}$ cells $\mathrm{m}^{-3}$ & $10^{15}$ cells $\mathrm{m}^{-3}$ \\
\hline$c^{e q m}$ & $\begin{array}{l}\text { Average concentration of EGF } \\
\text { i.e. concentration of } \\
\text { typical stimulus }\end{array}$ & $7 \times 10^{-10} \mathrm{M}$ & $7 \times 10^{-10} \mathrm{M}$ & $7 \times 10^{-10} \mathrm{M}$ \\
\hline$k$ & $\begin{array}{l}\text { Ratio of diffusion } \\
\text { coefficient of } \mathrm{P} \text { and } \mathrm{Q} \text { cell }\end{array}$ & 0.15 & 0.15 & 0.55 \\
\hline$\alpha_{*}$ & $\begin{array}{l}\text { Coefficient for Q cell } \\
\text { diffusion }\end{array}$ & 0.007 & 0.006 & 0.0030 \\
\hline$\overline{\alpha_{*}}$ & $\begin{array}{c}\text { Coefficient for stimulus } \\
\text { Q cell diffusion } \\
\text { diffusion }\end{array}$ & 0.007 & 0.006 & 0.0055 \\
\hline$\rho *$ & $\begin{array}{c}\text { Coefficient for P cell mitosis } \\
\& \text { differentiation }\end{array}$ & 0.05 & 0.05 & 0.25 \\
\hline$\overline{\rho *}$ & $\begin{array}{l}\text { Coefficient for stimulus } \\
\text { dependent P cell mitosis } \\
\& \text { differentiation }\end{array}$ & 0.95 & 0.95 & 0.75 \\
\hline$P_{*}^{\prime}$ & $\begin{array}{l}\text { With } a_{*} \& \sigma_{*} \text { below, } \\
\text { controls levels } \\
\text { of P cells }\end{array}$ & 0.20 & 0.18 & 0.08 \\
\hline $\begin{array}{l}\chi_{*}=\chi_{*}^{\text {healthy eye }} \\
-\end{array}$ & $\begin{array}{l}\text { Coefficient for stem cell } \\
\text { production of } \mathrm{P} \text { cells }\end{array}$ & 0.0 & 0.0010 & 0.0010 \\
\hline$\overline{\chi_{*}}=\bar{\chi}_{*}^{\text {healthy eye }}$ & $\begin{array}{l}\text { Coefficient for stimulus } \\
\text { dependent stem cell } \\
\text { production of P cells }\end{array}$ & 0.2 & 0.0040 & 0.0040 \\
\hline$\sigma_{*}$ & $\begin{array}{l}\text { Desquamation rate } \\
\text { of Q cells }\end{array}$ & 0.20 & 0.050 & 0.037 \\
\hline$D_{\text {stim }^{*}}$ & Stimulus diffusion & 200 & 120 & 65 \\
\hline$A_{*}$ & $\begin{array}{l}\text { Strength of tear } \\
\text { stimulus source }\end{array}$ & $\mu / 4+\delta$ & $\mu / 4+\delta$ & $\mu / 4+\delta$ \\
\hline$\mu_{*}$ & $\begin{array}{l}\text { Strength of stimulus } \\
\text { internalisation by cells }\end{array}$ & $1.2 * 10^{4}$ & $4.8 * 10^{3}$ & $4.8 * 10^{3}$ \\
\hline$c_{*}$ & $\begin{array}{l}\text { Cf. cell-chemical } \\
\text { internalisation by cells }\end{array}$ & 3.0 & 3.0 & 3.0 \\
\hline$\delta$ & $\begin{array}{c}(\ln 2) /(\text { half-life } \\
\text { of EGF (i.e. typical } \\
\text { stimulus)) }\end{array}$ & $\begin{array}{l}0.7 \mathrm{hr}^{-1} \\
\delta_{*}=25\end{array}$ & $\begin{array}{l}1.5 \mathrm{hr}^{-1} \\
\delta_{*}=11\end{array}$ & $\begin{array}{l}0.9 \mathrm{hr}^{-1} \\
\delta_{*}=19\end{array}$ \\
\hline$\psi_{*} \equiv B_{*}(0)$ & $\begin{array}{l}\text { Wound bed } \\
\text { source term }\end{array}$ & $\begin{aligned} & 1.7 \mathrm{~A} \\
= & 5.1 \times 10^{3}\end{aligned}$ & $\begin{aligned} & 1.0 \mathrm{~A} \\
= & 1.2 \times 10^{3}\end{aligned}$ & $\begin{aligned} & 1.0 \mathrm{~A} \\
= & 1.2 \times 10^{3}\end{aligned}$ \\
\hline \multicolumn{2}{|l|}{$\begin{array}{l}n=\text { Controls cell's } \\
\text { sensitivity to stimulus }\end{array}$} & 2 & 2 & 2 \\
\hline$a$ & Controls P & - & 0.22 & 0.4 \\
\hline$\vartheta$ & Controls P cell & - & 1.6 & 1.6 \\
\hline$P^{0}\left\{P^{1}\right\}$ & $\begin{array}{c}\text { Controls P cell } \\
\text { juxtacrine mechanism }\end{array}$ & - & - & $\begin{array}{l}(1 / 3)\{(1 / 5)\} \times 0.2 \times \\
\left.\left(P^{\text {eqm }}+Q^{\text {eqm }}\right)\right|_{r=0}\end{array}$ \\
\hline
\end{tabular}

Defining $V$ as the local radial velocity, we require the convective flux $V(P+Q)$ to be of the same order of magnitude as the flux of cells in the model, which yields

$$
(\alpha+\bar{\alpha})\left(\frac{\partial Q}{\partial r}+\kappa \frac{\partial P}{\partial r}\right) \sim V(P+Q) .
$$

Integrating over the radial variable, on use of the integral mean value theorem, yields

$$
(\alpha+\bar{\alpha})[(Q(R)-Q(0))+\kappa(P(R)-P(0))]
$$


for some $\zeta \in[0, R]$. The variation in the $P$ cells is much smaller than that of the $Q$ cells, and is also multiplied by $k<1$, and thus can be ignored at this level of approximation. Hence, on non-dimensionalisation, we have

$$
\left(\alpha_{*}+\bar{\alpha}_{*}\right) \sim \frac{\left(Q_{*}\left(\zeta_{*}\right)+P_{*}\left(\zeta_{*}\right)\right)}{[Q(1)-Q(0)]} \frac{T}{R} V_{a v}
$$

for some $\zeta_{*} \in[0,1]$. Now $\left(Q_{*}\left(\zeta_{*}\right)+P_{*}\left(\zeta_{*}\right)\right) /$ $[Q(1)-Q(0)]$ is an order one quantity, as

$$
P_{*}\left(\zeta_{*}\right) \ll Q_{*}(0) \leqslant \mathrm{Q}_{*}\left(\zeta_{*}\right) \leqslant \mathrm{Q}_{*}(1) \sim 2 Q_{*}(0)
$$

by the constraints of Section 4.1, and by the fact one would expect far fewer proliferative cells than quiescent cells, as the former tend to be localised in the basal layers. Thus we have, finally, the very simple relation

$$
\left(\alpha_{*}+\bar{\alpha}_{*}\right) \sim \frac{T}{R} V_{a v}=0.007
$$

which gives the order of magnitude required for consistency with Buck's experiment (Buck, 1985). (This corresponds to a non-dimensionalised diffusion coefficient of $10^{-8} \mathrm{~cm}^{2} \mathrm{~s}^{-1}$ ).

Data on stem cells is scarce due to the fact there is not, at present, a marker for corneal epithelial stem cells [though there are some potential markers (Zieske et al., 1992)]. However, it is known that the epithelial stem cell has a cell cycle time of greater than 14 days (Kruse, 1994), and it is commonly perceived that they exist only in the basal layer of the limbus (Tseng, 1989). This yields an upper bound for $\chi_{*}+\bar{\chi}_{*}$ as follows. On a time-scale greater than 14 days, the stem cells, which at most represent the basal layer of the limbus (i.e. a non-dimensionalised cell density of $\leqslant 0.1)$ produce one priliferative cell each. Hence, to be consistent with the above, one would require

$$
\chi^{*}+\bar{\chi}^{*}<\frac{1}{14} \times 0.1=7 \times 10^{-3} .
$$

There is a wide range of data on desquamation rates ranging from about 10-100 cells per cornea per minute (Rei \& Wilson, 1996), which corresponds to non-dimensionalised values between 0.008 and 0.08 [though the lower values are considered more appropriate in Rei \& Wilson (1996)]. Also note that, for a given value of $\alpha_{*}, \sigma_{*}$ and $P_{*}^{\prime}$ are heavily constrained by the requirements of Section 4.1 .
The value of $D_{\text {stim }}$ may be estimated from theoretical considerations to be about $10^{-6} \mathrm{~cm}^{2} \mathrm{~s}^{-1}$ (Berg \& Van Hippel, 1985), which yields non-dimensionalised estimates of order one. However, it is likely that any chemical stimulus may be transported by many means other than simply tear fluid diffusion within the cornea. Such mechanisms could include, for example, convection in the tear fluid flows and mixing due to blinking. Hence much larger values for $D_{\text {stim }}$ may realistically be used in the models above, and indeed are.

The value of $A_{*}$ is taken to be $\mu_{*} / 4+\delta_{*}$ for consistency [as in Dale et al. (1994a, b)], so that the non-dimensionalised central corneal stimulus has the value of 1.0. The estimation of $\mu_{*}$ is performed in Dale et al. (1994a, b), where for the non-dimensionalisation of this paper, one finds that $\mu_{*} \sim 2.8 \times 10^{3}$. The parameter $c_{*}$ is estimated as in Dale et al. (1994a, b), and is found to be roughly 3.0. In the papers Dale et al. $(1994 \mathrm{a}, \mathrm{b})$, it is speculated that $\psi_{*} \equiv B_{*}(0) \sim A_{*}$, though one may take $\psi_{*}$ to be significantly larger without contradiction of any experimental data, though this is not required for the models in this paper.

The half-life of a typical chemical stimulus yields an estimate for $\delta$. As the in vivo half-life of EGF is of the order of $1 \mathrm{~h}$ (Chan et al., 1991), this indicates that one should expect that $\delta \sim 1 \mathrm{hr}^{-1}$ which is true of the parameter values used.

The parameters $P^{1}$ and $P^{0}$ are chosen in accordance with their interpretation of juxtacrine mechanism controls. $P^{1}$ is chosen to be $(1 / 3) \times 0.2 \times\left.\left(P^{e q m}+Q^{e q m}\right)\right|_{r=0}$. Recall from Section 6 that this is one-third the cell density of a single cell layer, as $\left.\left(P^{e q m}+Q^{e q m}\right)\right|_{r=0}$ refers to the equilibrium cell density at the corneal centre, which is five cell layers thick. We take this for $P^{1}$ as for a hexagonal pattern of cells [i.e. a typical arrangement of epithelial cells (Wright \& Alison, 1984) it is a straightforward exercise to label one in three cells with no labelled cells touching. For such a hexagonal pattern of cells, consider the following. Each cell communicates with six neighbours, two of which are in a "forward" direction (with respect to some line in the plane of the epithelium, for example the edge of a wound bed), two are in a "backward" direction 
and two are in "side directions". Heuristically, a critical point where juxtacrine signalling becomes impaired is when the expectation for the number of neighbouring cells in the "side" or "forward" directions drops below unity. Naively, this occurs at the point when one in four cells of a single cell layer is proliferative, as a cell has four neighbours which are either "forward" or "side". We take $P^{0}$ to be below this naive critical point, and it corresponds to only one in five cells being proliferative.

The value of $\alpha_{*}-\bar{\alpha}_{*}$ is constrained to within an order of magnitude by requiring that the model wound healing does not occur on too slow or too fast a time-scale. The parameter $\alpha *$ cannot be estimated from kinetic data, which is normally expressed in terms of S-phase data. Consequently it is chosen in the manner outlined at the start of this section. Similarly for the parameters $\kappa, n, \vartheta_{*}, \rho_{*}-\bar{\rho}_{*}$, and $\chi_{*}-\bar{\chi}_{*}$.

\section{APPENDIX B}

\section{A Note on the Effect of Geometry on the Modelling of the Mitotic Rate}

It should be noted that considering a Taylor series expansion of the spatial dependence for the integrand in (1) yields a function of the form

mitotic rate $(r, t)=$

$$
\begin{aligned}
& \left(\left(\frac{100 \%}{\text { Total Cell Density }(r, t)}\right)\right. \\
& \left.\times \int_{t-4 \mathrm{hr}}^{t} \mathrm{~d} t^{\prime} \mathfrak{M}\left(r, t^{\prime}\right)\right)(1+O(\epsilon))
\end{aligned}
$$

For most 1-D coordinate systems $\epsilon$ takes the form

$$
\begin{array}{r}
\epsilon \sim \frac{1}{3} O\left[\Delta ^ { 2 } \left[\frac{\left(a \nabla^{2}-b \frac{\partial^{2}}{\partial^{2} r}\right) \mathfrak{M}}{\mathfrak{M}}\right.\right. \\
\left.\left.-\frac{\left(a \nabla^{2}-b \frac{\partial^{2}}{\partial^{2} r}\right)(\text { Total cell density })}{\text { Total cell density }}\right]\right]
\end{array}
$$

$\sim \frac{1}{3} O\left[\left(\frac{\text { Domain size }}{32}\right)^{2}\left[\frac{\left(a \nabla^{2}-b \frac{\partial^{2}}{\partial^{2} r}\right) \mathfrak{M}}{\mathfrak{M}}\right.\right.$

$$
\left.-\frac{\left(a \nabla^{2}-b \frac{\partial^{2}}{\partial^{2} r}\right)(\text { Total cell density })}{\text { Total cell density }}\right],
$$

where $a, b \leqslant O(1)$. For cartesians, $a=1, b=0$, with $r$ the spatial coordinate, whereas for axisymmetric cylindrical polars $a=1, b=0.5$, with $r$ the radial coordinate and for axisymmetric spherical polars $a=1, b=0.5$ with $r$ the meridional angle.

For all the results presented in this paper, it has been numerically confirmed that ignoring terms of $O(\epsilon)$ for a cylindrical polar formulation results in a relative error normally less than $0.5 \%$, and always less than $\sim 3 \%$ (results not shown). Such small errors are due to the factor of $\left(1 /\left(3 \times(32)^{2}\right)\right)$ in the expression for $\epsilon$ above. Consequently, one may reasonably speculate that geometrical factors do not appear to be important for the 1-D modelling of cell kinetics based on (1), though this can only be easily demonstrated by a posteriori observation, due to the potential ability of the terms in eqn (B.2) to blow up at small $\mathfrak{M}$ or small cell densities. The above observation is consistent with the numerical observation that geometry is not an important factor in the CEWH modelling of wound healing speeds (Dale et al., 1994b; Gaffney et al., 1997).

Also, it is useful to note that working with the approximation obtained by assuming $\epsilon=0$ in eqn (B.1), rather than the exact expression (1) which yields eqn (6) for the coordinate system used in this paper, leads to a substantial speed-up of the computer simulations, for a relatively small error. Hence preliminary studies are often best performed using such an approximation. 
For most 1-D coordinate systems $\epsilon$ takes the form

$$
\begin{gathered}
\epsilon \sim \frac{1}{3} O\left[\Delta ^ { 2 } \left[\frac{\left(a \nabla^{2}-b \frac{\partial^{2}}{\partial^{2} r}\right) \mathfrak{M}}{\mathfrak{M}}\right.\right. \\
\left.\left.-\frac{\left(a \nabla^{2}-b \frac{\partial^{2}}{\partial^{2} r}\right)(\text { Total cell density })}{\text { Total cell density }}\right]\right]
\end{gathered}
$$

$\sim \frac{1}{3} O\left[\left(\frac{\text { Domain size }}{32}\right)^{2}\left[\frac{\left(a \nabla^{2}-b \frac{\partial^{2}}{\partial^{2} r}\right) \mathfrak{M}}{\mathfrak{M}}\right.\right.$

$$
\left.\left.-\frac{\left(a \nabla^{2}-b \frac{\partial^{2}}{\partial^{2} r}\right)(\text { Total cell density })}{\text { Total cell density }}\right]\right] \text {, }
$$

where $a, b \leqslant O(1)$. For cartesians, $a=1, b=0$, with $r$ the spatial coordinate, whereas for axisymmetric cylindrical polars $a=1, b=0.5$, with $r$ the radial coordinate and for axisymmetric spherical polars $a=1, b=0.5$ with $r$ the meridional angle.

For all the results presented in this paper, it has been numerically confirmed that ignoring terms of $O(\epsilon)$ for a cylindrical polar formulation results in a relative error normally less than $0.5 \%$, and always less than $\sim 3 \%$ (results not shown). Such small errors are due to the factor of $\left(1 /\left(3 \times(32)^{2}\right)\right)$ in the expression for $\epsilon$ above. Consequently, one may reasonably speculate that geometrical factors do not appear to be important for the 1-D modelling of cell kinetics based on (1), though this can only be easily demonstrated by a posteriori observation, due to the potential ability of the terms in eqn (B.2) to blow up at small $\mathfrak{M}$ or small cell densities. The above observation is consistent with the numerical observation that geometry is not an important factor in the CEWH modelling of wound healing speeds (Dale et al., 1994b; Gaffney et al., 1997).

Also, it is useful to note that working with the approximation obtained by assuming $\epsilon=0$ in eqn (B.1), rather than the exact expression (1) which yields eqn (6) for the coordinate system used in this paper, leads to a substantial speed-up of the computer simulations, for a relatively small error. Hence preliminary studies are often best performed using such an approximation. 